\title{
FOX PAIRINGS AND GENERALIZED DEHN TWISTS
}

\author{
GWÉNAËL MASSUYEAU AND VLADIMIR TURAEV
}

\begin{abstract}
We introduce a notion of a Fox pairing in a group algebra and use Fox pairings to define automorphisms of the Malcev completions of groups. These automorphisms generalize to the algebraic setting the action of the Dehn twists in the group algebras of the fundamental groups of surfaces. This work is inspired by the Kawazumi-Kuno generalization of the Dehn twists to non-simple closed curves on surfaces.
\end{abstract}

\section{INTRODUCTION}

There is a simple and well-known construction producing families of automorphisms of modules from bilinear forms. Given a module $H$ over a commutative ring $\mathbb{K}$ and a bilinear form $\cdot: H \times H \rightarrow \mathbb{K}$, one associates with any isotropic vector $a \in H$ and any $k \in \mathbb{K}$ a transvection $H \rightarrow H$ carrying each $h \in H$ to $h+k(a \cdot h) a$. We introduce in this paper a group-theoretic version of transvections. Note that any group $\pi$ has a Malcev completion $\hat{\pi}=\hat{\pi}^{\mathbb{K}}$ formed by the group-like elements of the Hopf algebra $\widehat{\mathbb{K}[\pi}]$ which is the fundamental completion of the group algebra $\mathbb{K}[\pi]$, see $\mathrm{Qu}$. Our main construction starts with a group $\pi$ and a certain bilinear form, a Fox pairing, in $\mathbb{K}[\pi]$ and produces a family of group automorphisms of $\hat{\pi}$ which are in many respects similar to transvections.

Our original motivation comes from the study of diffeomorphisms of surfaces. Recall that simple closed curves on a connected oriented surface $\Sigma$ give rise to diffeomorphisms $\Sigma \rightarrow \Sigma$ called the Dehn twists. The Dehn twists induce group automorphisms of $\pi_{1}(\Sigma)$ and algebra automorphisms of $\mathbb{K}\left[\pi_{1}(\Sigma)\right]$ and $\left.\widehat{\mathbb{K}} \widehat{\left[\pi_{1}(\Sigma)\right.}\right]$. When $\Sigma$ is compact and $\partial \Sigma$ is a circle, N. Kawazumi and Y. Kuno KK1 generalized the action of the Dehn twists on $\left.\widehat{\mathbb{K}} \widehat{\left[\pi_{1}(\Sigma)\right.}\right)$ to arbitrary (not necessarily simple) loops on $\Sigma$. Their definition uses so-called symplectic expansions of $\pi_{1}(\Sigma)$, see [Ma. The present paper arose from our desire to avoid the use of symplectic expansions and to generalize the Kawazumi-Kuno automorphisms to all oriented surfaces. One simplification achieved here consists in replacing algebra automorphisms of the completed group algebras by group automorphisms of the Malcev completions.

The key ingredient in our approach is the homotopy intersection form on surfaces introduced by the second named author in [Tu1]. A version of this form was implicit already in the work of C. Papakyriakopoulos [Pa] who studied Reidemeister's equivariant intersection pairings on surfaces. Axiomatizing the homotopy intersection form, we introduce a notion of a Fox pairing in the group algebra $A=\mathbb{K}[\pi]$ of an arbitrary group $\pi$. Let $I \subset A$ be the fundamental ideal of $A$ defined as the kernel of the augmentation homomorphism aug : $A \rightarrow \mathbb{K}$ carrying $\pi \subset A$ to 1 . A Fox pairing in $A$ is a $\mathbb{K}$-bilinear pairing $\eta: A \times A \rightarrow A$ such that $1 \in A$ lies in both left and right annihilators and the restriction of $\eta$ to $I \times I$ is left $A$-linear in the first variable and right $A$-linear in the second variable. Similar pairings were studied in 
Pa and Tu1. A Fox pairing $\eta$ determines a $\mathbb{K}$-valued bilinear form ${ }^{\prime} \eta$ on $H_{1}(\pi ; \mathbb{K})$ which generalizes the usual intersection form in the homology of a surface.

The general algebraic framework for Dehn-type twists on a group $\pi$ involves a choice of a commutative ring $\mathbb{K} \supset \mathbb{Q}$ and a choice of a Fox pairing $\eta$ in $A=\mathbb{K}[\pi]$ (more generally, one may start from a Fox pairing in $\widehat{A}=\lim A / I^{m}$ ). For every $a \in \hat{\pi}$ such that the homology class $[a] \in H_{1}(\pi ; \mathbb{K})$ satisfies $[a] \eta_{\eta}[a]=0$, we define a 1 -parameter family $\left(t_{k, a}\right)_{k \in \mathbb{K}}$ of automorphisms of $\hat{\pi}$ called the twists. The definition of the twists goes by exponentiating certain derivations of $\widehat{A}$ determined by $\eta$. Among properties of the twists $t_{k, a}$ established here, note that they depend only on the conjugacy class of $a$ and $t_{k, a^{-1}}=t_{k, a}$. Using the canonical homomorphism $\pi \rightarrow \hat{\pi}$ we can derive automorphisms of $\hat{\pi}$ from conjugacy classes in $\pi$.

Our main example concerns the surfaces. For a connected oriented surface $\Sigma$ with non-empty boundary, the group $\pi=\pi_{1}(\Sigma, *)$ with $* \in \partial \Sigma$ has a natural Fox pairing $\eta$ which is (essentially) the homotopy intersection form of [Tu1]. Here the form $\eta_{\eta}$ is skew-symmetric. Thus, any conjugacy class in $\hat{\pi}$ yields a 1-parameter family of automorphisms of $\hat{\pi}$ called the generalized Dehn twists. Every closed curve $C$ in $\Sigma$ represents a conjugacy class in $\pi$ and determines in this way a family $\left(t_{k, C}\right)_{k \in \mathbb{K}}$ of automorphisms of $\hat{\pi}$. We prove that if $C$ is simple (i.e., has no selfintersections), then $t_{1 / 2, C}$ is the automorphism of $\hat{\pi}$ induced by the Dehn twist about $C$. One interesting application is that for any integer $N \geq 2$, the action of the Dehn twist on $\hat{\pi}$ has a canonical $N$-th root $t_{1 / 2 N, C} \in$ Aut $\hat{\pi}$. We show that when $\Sigma$ is compact, $\partial \Sigma \cong S^{1}$, and $\mathbb{K}=\mathbb{Q}$, the Kawazumi-Kuno automorphism of $\widehat{\mathbb{K}[\pi]}$ determined by any closed curve $C$ in $\Sigma$ is the extension of $t_{1 / 2, C} \in$ Aut $\hat{\pi}$.

We obtain similar results for closed curves on surfaces without boundary, and without base point. In this case, the generalized Dehn twists belong to the outer automorphism group of $\hat{\pi}$.

The paper consists of the algebraic part, Sections 2 6, and the geometric part, Sections 7 10, In the algebraic part we introduce and study Fox pairings in group algebras and define the associated twists. In Sections 7 9, we recall the definition of the homotopy intersection forms on surfaces and define the generalized Dehn twists about curves. In Section 10 we show that for compact surfaces with boundary $S^{1}$ our definition is equivalent to the one due to N. Kawazumi and Y. Kuno. The paper ends with an appendix where we collect some classical identities for the logarithm and the exponential series frequently used in the paper.

Soon after appearance of this paper in the arXiv, N. Kawazumi and Y. Kuno informed us that they had obtained similar results for based oriented surfaces, see KK2.

Acknowledgement. The work of G. Massuyeau was partially supported by the French ANR research project ANR-08-JCJC-0114-01. The work of V. Turaev was partially supported by the NSF grant DMS-0904262. A part of this paper was written during a visit of the authors to the Centre for Quantum Geometry of Moduli Spaces, at Aarhus University in summer 2011; the authors thank QGM for hospitality.

\section{FOX PAIRINGS AND THE DERIVED FORMS}

We fix a group $\pi$ and a commutative ring $\mathbb{K}$. We use notation $A=\mathbb{K}[\pi]$, aug : $A \rightarrow \mathbb{K}$, and $I=\operatorname{Ker}($ aug) as above. In this section, we introduce and study 
Fox pairings in $A$. In particular, we derive from any such pairing a certain bilinear form $A \times A \rightarrow A$ which shall play a crucial role in the sequel.

2.1. Derivations and Fox derivatives. A derivation of a $\mathbb{K}$-algebra $L$ is a $\mathbb{K}$ linear homomorphism $d: L \rightarrow L$ such that $d(a b)=d(a) b+a d(b)$ for all $a, b \in L$. The derivations of $L$ form a Lie algebra $\operatorname{Der}(L)$ over $\mathbb{K}$ with Lie bracket $\left[d_{1}, d_{2}\right]=$ $d_{1} d_{2}-d_{2} d_{1}$.

We shall approach derivations of the algebra $A=\mathbb{K}[\pi]$ via the Fox calculus. Recall that a $\mathbb{K}$-linear map $\partial: A \rightarrow A$ is a left (resp. a right) Fox derivative if for all $a, b \in A$, we have $\partial(a b)=\partial(a) \operatorname{aug}(b)+a \partial(b)($ resp. $\partial(a b)=\partial(a) b+\operatorname{aug}(a) \partial(b))$. Any Fox derivative carries $1 \in A$ to 0 .

For example, for $e \in A$, the map $A \rightarrow A$ carrying any $a \in A$ to $(a-\operatorname{aug}(a)) e$ is a left Fox derivative and the map $A \rightarrow A$ carrying any $a \in A$ to $e(a-\operatorname{aug}(a))$ is a right Fox derivative.

Multiplying the values of a left Fox derivative on the right by an element of $A$ we obtain again a left Fox derivative. In this way, the left Fox derivatives form a right $A$-module denoted $D_{l}$. Similarly, the right Fox derivatives form a left $A$-module $D_{r}$. Restricting the derivatives to $I \subset A$, we obtain an isomorphism of $D_{l}$ (resp. $D_{r}$ ) onto the $\mathbb{K}$-module of $\mathbb{K}$-linear homomorphisms $I \rightarrow A$ that are $A$-linear on the left (resp. on the right). There is a canonical $\mathbb{K}$-linear isomorphism $D_{l} \simeq D_{r}$. It sends a left Fox derivative $\partial$ into the right Fox derivative carrying any $a \in A$ to $\overline{\partial(\bar{a})}$. Here and below the overbar denotes the canonical $\mathbb{K}$-linear involution in $A$ inverting the elements of $\pi$.

The following lemma produces derivations from Fox derivatives. First, a piece of notation. Any element $u$ of $A$ expands uniquely as $u=\sum_{x \in \pi} k_{x} x$ where $k_{x} \in \mathbb{K}$ and $k_{x}=0$ for all but a finite set of $x \in \pi$. For $v \in A$, set $v^{u}=\sum_{x \in \pi} k_{x} x^{-1} v x \in A$. It is clear that $v^{u}$ is $\mathbb{K}$-linear in both $u$ and $v$. Note the following obvious identities: for any $u, v \in A$ and $a \in \pi$,

$$
a v^{u a}=v^{u} a \text { and }(a v)^{a u}=(v a)^{u} .
$$

Lemma 2.1. Let $\partial: A \rightarrow A$ be a right Fox derivative and $v \in A$. Let $d: A \rightarrow A$ be the unique $\mathbb{K}$-linear map carrying any $a \in \pi$ to $a v^{\partial(a)}$. Then $d$ is a derivation. Similarly, if $\partial: A \rightarrow A$ is a left Fox derivative and $v \in A$, then the $\mathbb{K}$-linear map $A \rightarrow A$ carrying any $a \in \pi$ to $v^{\overline{\partial(a)}}$ a is a derivation.

Proof. It is enough to prove that $d(a b)=a d(b)+d(a) b$ for all $a, b \in \pi$. We have

$$
\begin{aligned}
d(a b) & =a b v^{\partial(a b)}=a b v^{\partial(a) b+\partial(b)} \\
& =a b v^{\partial(a) b}+a b v^{\partial(b)}=a v^{\partial(a)} b+a b v^{\partial(b)}=d(a) b+a d(b)
\end{aligned}
$$

where we use the first of the identities (2.1.1) with $a$ replaced by $b$ and $u=\partial(a)$. The second claim of the lemma is proved similarly.

2.2. Fox pairings. By a Fox pairing or shorter an F-pairing in $A$ we mean a $\mathbb{K}$ bilinear map $\eta: A \times A \rightarrow A$ which is a left Fox derivative with respect to the first variable and a right Fox derivative with respect to the second variable. Note for the record the product formulas

$$
\begin{aligned}
& \eta\left(a_{1} a_{2}, b\right)=\eta\left(a_{1}, b\right) \operatorname{aug}\left(a_{2}\right)+a_{1} \eta\left(a_{2}, b\right) \quad \text { for any } a_{1}, a_{2}, b \in A, \\
& \eta\left(a, b_{1} b_{2}\right)=\eta\left(a, b_{1}\right) b_{2}+\operatorname{aug}\left(b_{1}\right) \eta\left(a, b_{2}\right) \quad \text { for any } a, b_{1}, b_{2} \in A .
\end{aligned}
$$


Substituting $a_{1}=a_{2}=1$ in 2.2.1) we obtain $\eta(1, A)=0$. Similarly, $\eta(A, 1)=0$. It is clear that the restriction of $\eta$ to $I \times I \subset A \times A$ is linear with respect to left multiplication of the first variable by elements of $A$ and with respect to right multiplication of the second variable by elements of $A$. Therefore $\eta\left(I^{m}, I^{n}\right) \subset$ $I^{m+n-2}$ for all $m, n \geq 1$.

Similarly, we define a T-pairing in $A$ as a $\mathbb{K}$-bilinear map $\lambda: A \times A \rightarrow A$ which satisfies

$$
\begin{aligned}
& \lambda\left(a_{1} a_{2}, b\right)=\lambda\left(a_{1}, b\right) \operatorname{aug}\left(a_{2}\right)+a_{1} \lambda\left(a_{2}, b\right) \quad \text { for any } \quad a_{1}, a_{2}, b \in A \\
& \lambda\left(a, b_{1} b_{2}\right)=\lambda\left(a, b_{1}\right) \operatorname{aug}\left(b_{2}\right)+\lambda\left(a, b_{2}\right) \overline{b_{1}} \quad \text { for any } a, b_{1}, b_{2} \in A
\end{aligned}
$$

A T-pairing $\lambda$ determines an F-pairing $\eta$ by setting $\eta(a, b)=\lambda(a, b) b$ for all $a, b \in \pi$ and then extending to $A$ by linearity. This establishes a bijection between Tpairings and F-pairings and shows that these two notions are essentially equivalent. The T-pairings were first studied in $\mathrm{Pa}$. under the name of "biderivations" and in Tu1 under the name of " $\Delta$-forms".

The F-pairings in $A$ form a $\mathbb{K}$-module, $F(A)$, in the obvious way. Any $e \in A$ gives rise to an $\mathrm{F}$-pairing

$$
\eta_{e}(a, b)=(a-\operatorname{aug}(a)) e(b-\operatorname{aug}(b)) .
$$

We call such F-pairings inner. Further F-pairings may be obtained using the modules $D_{l}, D_{r}$ introduced in Section 2.1 and the $\mathbb{K}$-linear map $D_{l} \otimes_{A} D_{r} \rightarrow F(A)$ carrying $\partial \otimes \partial^{\prime} \in D_{l} \otimes_{A} D_{r}$ to the F-pairing $(a, b) \mapsto \partial(a) \partial^{\prime}(b)$.

2.3. The induced forms. Given an F-pairing $\eta: A \times A \rightarrow A$, we set $a \cdot \eta b=$ $\operatorname{aug}(\eta(a, b)) \in \mathbb{K}$ for any $a, b \in A$. It follows from (2.2.1) and (2.2.2) that the restriction of the resulting pairing $\eta: A \times A \rightarrow \mathbb{K}$ to $\pi \times \pi$ is additive in each variable. This restriction induces a $\mathbb{K}$-bilinear form $H \times H \rightarrow \mathbb{K}$ where $H=$ $H_{1}(\pi ; \mathbb{K})=\mathbb{K} \otimes_{\mathbb{Z}} \pi /[\pi, \pi]$. This form is denoted by the same symbol $\eta$ and is called the homological form induced by $\eta$.

The F-pairing $\eta$ gives rise to a right derived form $\sigma^{\eta}: A \times A \rightarrow A$ as follows. For any $a, b \in \pi$, set

$$
\sigma^{\eta}(a, b)=b a^{\eta(a, b)}=b \sum_{x \in \pi} k_{x} x^{-1} a x \in A
$$

where $\eta(a, b)=\sum_{x \in \pi} k_{x} x$ with $k_{x} \in \mathbb{K}$. Then extend the resulting map $\pi \times \pi \rightarrow A$ to $A \times A$ by $\mathbb{K}$-bilinearity. The left derived form is the $\mathbb{K}$-bilinear map ${ }^{\eta} \sigma: A \times A \rightarrow A$ carrying any pair $(a, b) \in \pi \times \pi$ to $b^{\overline{\eta(a, b)}} a$. The left and the right derived forms have similar properties. We will focus on the right derived form $\sigma^{\eta}$. When there is no ambiguity, it is called the derived form of $\eta$ and denoted $\sigma$.

We have $\operatorname{aug}(\sigma(a, b))=a \cdot \eta b$ for all $a, b \in A$. This follows from the definitions for $a, b \in \pi$ and extends to all $a, b$ by bilinearity. The equalities $\eta(1, A)=\eta(A, 1)=0$ imply that $\sigma(1, A)=\sigma(A, 1)=0$. We now state a few properties of $\sigma$.

Lemma 2.2. For any $a \in A$, the map $A \rightarrow A, b \mapsto \sigma(a, b)$ is a derivation of $A$.

Proof. This directly follows from the definitions and Lemma 2.1

Lemma 2.3. For any $m \geq 1$, we have $\sigma\left(I^{m}, A\right) \subset I^{m-1}$. 
Proof. The ideal $I^{m}$ is $\mathbb{K}$-linearly generated by the products

$$
\Pi=\left(a_{1}-1\right) \cdots\left(a_{m}-1\right)
$$

where $a_{1}, \ldots, a_{m} \in \pi$. It is enough to prove that $\sigma(\Pi, b) \in I^{m-1}$ for each such $\Pi$ and any $b \in \pi$. By the bilinearity of $\sigma$ and the property $\sigma(1, A)=0$,

$$
\sigma(\Pi, b)=\sum_{r=1}^{m} \sum_{1 \leq i_{1}<\cdots<i_{r} \leq m}(-1)^{m-r} \sigma\left(a_{i_{1}} \cdots a_{i_{r}}, b\right) .
$$

Observe that

$$
\eta\left(a_{i_{1}} \cdots a_{i_{r}}, b\right)=\sum_{s=1}^{r} a_{i_{1}} \cdots a_{i_{s-1}} \eta\left(a_{i_{s}}, b\right) .
$$

For all $i=1, \ldots, m$, we expand $\eta\left(a_{i}, b\right)=\sum_{x \in \pi} n_{x}^{i} x$ with $n_{x}^{i} \in \mathbb{K}$. Then

$$
\begin{aligned}
\sigma\left(a_{i_{1}} \cdots a_{i_{r}}, b\right) & =b\left(a_{i_{1}} \cdots a_{i_{r}}\right)^{\eta\left(a_{i_{1}} \cdots a_{i_{r}}, b\right)} \\
& =b \sum_{s=1}^{r}\left(a_{i_{1}} \cdots a_{i_{r}}\right)^{a_{i_{1}} \cdots a_{i_{s-1}} \eta\left(a_{i_{s}}, b\right)} \\
& =b \sum_{s=1}^{r} \sum_{x \in \pi} n_{x}^{i_{s}}\left(a_{i_{1}} \cdots a_{i_{s-1}} x\right)^{-1}\left(a_{i_{1}} \cdots a_{i_{r}}\right)\left(a_{i_{1}} \cdots a_{i_{s-1}} x\right) \\
& =b \sum_{s=1}^{r} \sum_{x \in \pi} n_{x}^{i_{s}} x^{-1} a_{i_{s}} a_{i_{s+1}} \cdots a_{i_{r}} a_{i_{1}} \cdots a_{i_{s-1}} x .
\end{aligned}
$$

Substituting this formula in the expansion of $\sigma(\Pi, b)$ above, we obtain that

$$
\sigma(\Pi, b)=b \sum_{x \in \pi} x^{-1} T_{x} x
$$

where $T_{x}$ denotes the triple sum

$$
\begin{aligned}
& \sum_{r=1}^{m} \sum_{1 \leq i_{1}<\cdots<i_{r} \leq m} \sum_{s=1}^{r}(-1)^{m-r} n_{x}^{i_{s}} a_{i_{s}} a_{i_{s+1}} \cdots a_{i_{r}} a_{i_{1}} \cdots a_{i_{s-1}} \\
& =\sum_{j=1}^{m} \sum_{\substack{0 \leq t \leq j-1 \\
0 \leq u \leq m-j}} \sum_{\substack{1 \leq k_{1}<\cdots<k_{t}<j \\
j<l_{1}<\cdots<l_{u} \leq m}}(-1)^{m-(t+u+1)} n_{x}^{j} a_{j} a_{l_{1}} \cdots a_{l_{u}} a_{k_{1}} \cdots a_{k_{t}} \\
& =\sum_{j=1}^{m} \sum_{\substack{0 \leq t \leq j-1 \\
0 \leq u \leq m-j}} \sum_{\substack{1 \leq k_{1}<\cdots<k_{t}<j \\
j<l_{1}<\cdots<l_{u} \leq m}}(-1)^{(j-1)-t}(-1)^{(m-j)-u} n_{x}^{j} a_{j} a_{l_{1}} \cdots a_{l_{u}} a_{k_{1}} \cdots a_{k_{t}} \\
& =\sum_{j=1}^{m} n_{x}^{j} a_{j}\left(a_{j+1}-1\right) \cdots\left(a_{m}-1\right)\left(a_{1}-1\right) \cdots\left(a_{j-1}-1\right) .
\end{aligned}
$$

Therefore $\sigma(\Pi, b) \in I^{m-1}$.

Lemma 2.4. For any $m, n \geq 1$, we have $\sigma\left(I^{m}, I^{n}\right) \subset I^{m+n-2}$. Moreover, for any $c_{1}, \ldots, c_{m}, d_{1}, \ldots, d_{n} \in I$, we have the following congruence modulo $I^{m+n-1}$ : $\sigma\left(c_{1} \cdots c_{m}, d_{1} \cdots d_{n}\right) \equiv \sum_{i=1}^{m} \sum_{j=1}^{n}\left(c_{i} \cdot \eta d_{j}\right) d_{1} \cdots d_{j-1}\left(c_{i+1} \cdots c_{m} c_{1} \cdots c_{i-1}\right) d_{j+1} \cdots d_{n}$. 
Proof. Set $c=c_{1} \cdots c_{m}$ and $d=d_{1} \cdots d_{n}$. Since $\sigma(c,-)$ is a derivation of $A$,

$$
\sigma(c, d)=\sum_{j=1}^{n} d_{1} \cdots d_{j-1} \sigma\left(c, d_{j}\right) d_{j+1} \cdots d_{n} .
$$

By Lemma 2.3. $\sigma\left(c, d_{j}\right) \in I^{m-1}$. This implies that $\sigma(c, d) \in I^{m+n-2}$ and proves the first claim of the lemma. We now prove the second claim. Since $I$ is $\mathbb{K}$-linearly spanned by the set $\{a-1\}_{a \in \pi}$, we can assume that $c_{i}=a_{i}-1$ for all $i=1, \ldots, m$ and some $a_{i} \in \pi$ and similarly $d_{j}=b_{j}-1$ for all $j=1, \ldots, n$ and some $b_{j} \in \pi$. The proof of Lemma 2.3 shows that for all $j$,

$$
\sigma\left(c, d_{j}\right)=\sigma\left(c, b_{j}\right) \equiv \sum_{i=1}^{m} \sum_{x \in \pi} n_{x}^{i j} c_{i+1} \cdots c_{m} c_{1} \cdots c_{i-1}\left(\bmod I^{m}\right)
$$

where we have expanded $\eta\left(a_{i}, b_{j}\right)=\sum_{x \in \pi} n_{x}^{i j} x$ for all $i$. The required formula follows now from (2.3.1) because $\sum_{x \in \pi} n_{x}^{i j}=a_{i} \cdot{ }_{\eta} b_{j}=c_{i} \cdot{ }_{\eta} d_{j}$.

Lemma 2.5. We have $\sigma(a b, c)=\sigma(b a, c)$ for any $a, b, c \in A$.

Proof. By the $\mathbb{K}$-bilinearity of $\sigma$, it is enough to consider the case where $a, b, c \in \pi$. We have

$$
\begin{aligned}
\sigma(a b, c) & =c(a b)^{\eta(a b, c)}=c(a b)^{\eta(a, c)+a \eta(b, c)} \\
& =c(a b)^{\eta(a, c)}+c(a b)^{a \eta(b, c)}=c(a b)^{\eta(a, c)}+c(b a)^{\eta(b, c)}
\end{aligned}
$$

where we use the second of the identities (2.1.1). The right-hand side is preserved under the permutation of $a$ and $b$.

2.4. Equivalence and transposition of F-pairings. We call two F-pairings in $A$ equivalent if their difference is an inner F-pairing in the sense of Section 2.2 Equivalent F-pairings induce the same homological form and the same (left and right) derived forms.

Every F-pairing $\eta$ in $A$ determines a transposed F-pairing $\bar{\eta}$ in $A$ by

$$
\bar{\eta}(a, b)=a \overline{\eta(b, a)} b
$$

for all $a, b \in \pi$. The transposition of F-pairings is involutive and compatible with the equivalence. The left derived form ${ }^{\eta} \sigma$ of an F-pairing $\eta$ can be computed from the right derived form $\sigma^{\bar{\eta}}$ of $\bar{\eta}$ by ${ }^{\eta} \sigma(a, b)=\sigma^{\bar{\eta}}(b, a)$ for all $a, b \in A$.

We call an F-pairing $\eta$ weakly skew-symmetric if the F-pairing $\eta+\bar{\eta}$ is inner. Then ${ }^{\eta} \sigma(a, b)=-\sigma^{\eta}(b, a)$ for all $a, b \in A$.

2.5. Remarks. 1. It is easy to describe Fox derivatives and Fox pairings in $A$ when $\pi$ is a free group of finite rank $n$ with basis $\left\{x_{i}\right\}_{i=1}^{n}$. Then any $a \in A$ expands uniquely as the $\operatorname{sum} \operatorname{aug}(a)+\sum_{i} a_{i}\left(x_{i}-1\right)$ with $a_{i} \in A$. For all $i$, the map $A \rightarrow A, a \mapsto a_{i}$ is a left Fox derivative denoted $\partial_{i}$. Also, any $a \in A$ expands uniquely as the $\operatorname{sum} \operatorname{aug}(a)+\sum_{i}\left(x_{i}-1\right) a^{i}$ with $a^{i} \in A$ and for all $i$, the map $A \rightarrow A, a \mapsto a^{i}$ is a right Fox derivative denoted $\partial^{i}$. For any $n \times n$ matrix $\left(\eta_{i, j}\right)$ over $A$ there is a unique F-pairing $\eta$ in $A$ such that $\eta\left(x_{i}, x_{j}\right)=\eta_{i, j}$ for all $i, j$. This pairing is computed by $\eta(a, b)=\sum_{i, j} \partial_{i}(a) \eta_{i, j} \partial^{j}(b)$ for $a, b \in A$. All F-pairings in $A$ arise in this way.

2. Lemma 2.5 implies that $\sigma$ is invariant under conjugations of the first variable: $\sigma\left(c a c^{-1}, b\right)=\sigma(a, b)$ for any $a, b \in A$ and $c \in \pi$. This fact has a weaker analogue for the second variable. Let $\check{\pi}$ be the set of conjugacy classes of $\pi$ and let $\mathbb{K}[\check{\pi}]$ be 
the free $\mathbb{K}$-module with basis $\check{\pi}$. Let $q: A \rightarrow \mathbb{K}[\check{\pi}]$ be the $\mathbb{K}$-linear map induced by the projection $\pi \rightarrow \check{\pi}$. Then the composition $q \sigma: A \times A \rightarrow \mathbb{K}[\check{\pi}]$ is invariant under conjugations in both variables. The invariance under conjugations of the second variable can be proven similarly to Lemma 2.5 or can be deduced from Lemma 2.2 and the following general fact: for any derivation $d: A \rightarrow A$ and any $b, c \in \pi$, we have $q d\left(c b c^{-1}\right)=q d(b)$. We conclude that $q \sigma$ induces a $\mathbb{K}$-bilinear form $\mathbb{K}[\check{\pi}] \times \mathbb{K}[\check{\pi}] \rightarrow \mathbb{K}[\check{\pi}]$.

\section{Completions}

We consider the fundamental completion $\hat{A}$ of the group algebra $A$ and extend to $\hat{A}$ the definitions and constructions of Section 2 We keep the notation of Section 2

3.1. The algebra $\hat{A}$. The powers of the fundamental ideal $I \subset A=\mathbb{K}[\pi]$ form a filtration $A=I^{0} \supset I \supset I^{2} \supset \cdots$ by two-sided ideals. Let $\hat{A}=\lim _{\longleftarrow} A / I^{m}$ be the completion of $A$ with respect to this filtration. Clearly, $\hat{A}$ is a unital $\mathbb{K}$-algebra. It is called the fundamental completion of $A$. The projections $\left\{A \rightarrow A / I^{m}\right\}_{m}$ define an algebra homomorphism $A \rightarrow \hat{A}$ denoted by $\iota$. Generally speaking, this homomorphism is not injective.

For any integer $m \geq 0$ denote by $\hat{A}_{m}$ the kernel of the projection $\hat{A} \rightarrow A / I^{m}$. Clearly, $\hat{A}=\hat{A}_{0} \supset \hat{A}_{1} \supset \hat{A}_{2} \supset \cdots$ is a filtration of $\hat{A}$ by two-sided ideals, and the completion of $\hat{A}$ with respect to this filtration gives the same algebra $\hat{A}$. Note for the record that $\hat{A}_{m} \hat{A}_{n} \subset \hat{A}_{m+n}$ and $\iota\left(I^{m}\right) \subset \hat{A}_{m}$ for all $m, n \geq 0$.

The algebra $\hat{A}$ has a canonical augmentation aûg : $\hat{A} \rightarrow \mathbb{K}$ defined as the projection $\hat{A} \rightarrow \hat{A} / \hat{A}_{1} \simeq A / I$ followed by aug : $A / I \stackrel{\widetilde{ }}{\rightarrow} \mathbb{K}$.

To study comultiplications in $\hat{A}$, we endow the algebra $A \otimes A$ with the filtration

$$
A \otimes A=(A \otimes A)_{0} \supset(A \otimes A)_{1} \supset(A \otimes A)_{2} \supset \cdots
$$

whose for all $m \geq 0$, the $m$-th term is the two-sided ideal

$$
(A \otimes A)_{m}=\sum_{p=0}^{m} I^{p} \otimes I^{m-p} .
$$

Let $A \hat{\otimes} A$ be the completion of $A \otimes A$ with respect to this filtration:

$$
A \hat{\otimes} A=\lim _{\longleftarrow}(A \otimes A) /(A \otimes A)_{m} .
$$

The algebra $A \hat{\otimes} A$ is endowed with the filtration whose $m$-th term $(A \hat{\otimes} A)_{m}$ is the kernel of the projection $A \hat{\otimes} A \rightarrow(A \otimes A) /(A \otimes A)_{m}$ for all $m \geq 0$. For any $a, b \in A$, the image of $a \otimes b \in A \otimes A$ under the natural homomorphism $A \otimes A \rightarrow A \hat{\otimes} A$ is denoted $a \hat{\otimes} b$. One similarly defines filtered algebras $A^{\hat{\otimes} 3}=A \hat{\otimes} A \hat{\otimes} A, A^{\hat{\otimes}} 4=$ $A \hat{\otimes} A \hat{\otimes} A \hat{\otimes} A$, etc. Applying these definitions to $\hat{A}$ rather than $A$, we obtain the same filtered algebras: it is easy to check that $\iota: A \rightarrow \hat{A}$ induces an isomorphism of filtered algebras $A^{\hat{\otimes} n} \rightarrow \hat{A}^{\hat{\otimes} n}$ for all $n \geq 2$.

The algebra $A$ has a standard comultiplication $\Delta: A \rightarrow A \otimes A$ carrying any $x \in \pi$ to $x \otimes x$. The formulas

$$
\Delta(x-1)=x \otimes x-1 \otimes 1=x \otimes(x-1)+(x-1) \otimes x-(x-1) \otimes(x-1)
$$

show that $\Delta(I) \subset A \otimes I+I \otimes A$. Therefore $\Delta$ is compatible with the filtrations in the sense that $\Delta\left(I^{m}\right) \subset(A \otimes A)_{m}$ for all $m \geq 0$. Hence $\Delta$ induces an algebra homomorphism $\hat{\Delta}: \hat{A} \rightarrow A \hat{\otimes} A=\hat{A} \hat{\otimes} \hat{A}$. The algebra $\hat{A}$ with "comultiplication" 
$\hat{\Delta}$ and counit aûg is a complete Hopf algebra in the sense of Quillen $\mathrm{Qu}$. The antipode in $\hat{A}$ is the involution of $\hat{A}$ induced by the involution $a \mapsto \bar{a}$ of $A$.

For example, if $\pi$ is a free group, then the homomorphism $\iota: A \rightarrow \hat{A}$ is injective, and one usually uses it to treat $A$ as a subalgebra of $\hat{A}$. Given a basis $\left\{x_{i}\right\}_{i}$ of $\pi$, one can identify $\hat{A}$ with the algebra of formal power series in (non-commuting) variables $\left\{X_{i}\right\}_{i}$ with coefficients in $\mathbb{K}$, see MKS. One way to fix such an identification is to require that $x_{i}=1+X_{i}$ and $x_{i}^{-1}=1-X_{i}+X_{i}^{2}-\cdots$ for all $i$. Then the ideal $\hat{A}_{m} \subset \hat{A}$ with $m \geq 1$ corresponds to the ideal of formal power series containing no monomials of degree $<m$.

3.2. The group $\hat{\pi}$. Let $\hat{\pi}=\hat{\pi}^{\mathbb{K}}$ be the group-like part of $\hat{A}$. More precisely,

$$
\hat{\pi}=\{a \in \hat{A}: \hat{\Delta}(a)=a \hat{\otimes} a \text { and } a \neq 0\} .
$$

This is a subgroup of the multiplicative group $1+\hat{A}_{1}$. We endow $\hat{\pi}$ with the canonical filtration $\hat{\pi}=\hat{\pi}_{(1)} \supset \hat{\pi}_{(2)} \supset \hat{\pi}_{(3)} \supset \cdots$ where $\hat{\pi}_{(m)}=\hat{\pi} \cap\left(1+\hat{A}_{m}\right)$ for all $m \geq 1$. It is easy to see that $\hat{\pi}_{(m)}$ is a normal subgroup of $\hat{\pi}$ and $\left[\hat{\pi}_{(m)}, \hat{\pi}_{(n)}\right] \subset \hat{\pi}_{(m+n)}$ for all $m, n \geq 1$. The algebra homomorphism $\iota: A \rightarrow \hat{A}$ restricts to a group homomorphism $\pi \rightarrow \hat{\pi}$ that carries the lower central series $\pi=\pi_{1} \supset \pi_{2} \supset \cdots$ of $\pi$ into the canonical filtration of $\hat{\pi}$. We call $\hat{\pi}$ the Malcev completion of $\pi$ over $\mathbb{K}$. In the case $\mathbb{K}=\mathbb{Q}$, there is an isomorphism $\hat{\pi} \simeq \lim \left(\left(\pi / \pi_{m}\right) \otimes \mathbb{Q}\right)$ where $\left(\pi / \pi_{m}\right) \otimes \mathbb{Q}$ is the standard Malcev completion of the nilpotent group $\pi / \pi_{m}$, i.e. its uniquely divisible closure. For more on the Malcev completion, see Qu, J].

Lemma 3.1. If $\mathbb{Q} \subset \mathbb{K}$, then $\hat{\pi} / \hat{\pi}_{(2)} \simeq H_{1}(\pi ; \mathbb{K})$.

Proof. There is a $\mathbb{K}$-linear isomorphism $I / I^{2} \simeq H_{1}(\pi ; \mathbb{K})$ carrying $k(g-1)\left(\bmod I^{2}\right)$ to $k[g] \in H_{1}(\pi ; \mathbb{K})$ for all $k \in \mathbb{K}$ and $g \in \pi$. (The inverse isomorphism is induced by the group homomorphism $g \mapsto g-1\left(\bmod I^{2}\right)$ from $\pi$ to the abelian group $I / I^{2}$.) The formula $\psi(a)=a-1\left(\bmod \hat{A}_{2}\right)$ defines a homomorphism $\psi$ from $\hat{\pi}$ to the abelian group $\hat{A}_{1} / \hat{A}_{2} \simeq I / I^{2}$. By the definition of $\hat{\pi}_{(2)}$, we have $\operatorname{Ker} \psi=\hat{\pi}_{(2)}$. It remains to show that $\psi$ is onto. Since $\mathbb{Q} \subset \mathbb{K}$, the logarithmic and exponential series define mutually inverse bijections between $\hat{\pi}$ and the primitive part of $\hat{A}$ :

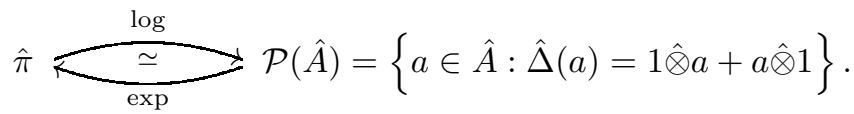

The set $\mathcal{P}(\hat{A})$ is a Lie $\mathbb{K}$-subalgebra of $\hat{A}_{1}$ (it is sometimes called the Malcev Lie algebra of $\pi)$. Let $g \in \pi$ and set $g^{\prime}=\iota(g) \in \hat{\pi}$. Then $\log \left(g^{\prime}\right) \in \mathcal{P}(\hat{A})$. For any $k \in \mathbb{K}$, we have $k \log \left(g^{\prime}\right) \in \mathcal{P}(\hat{A})$ and $a_{k}=\exp \left(k \log \left(g^{\prime}\right)\right) \in \hat{\pi}$. Observe that $a_{k} \equiv 1+k\left(g^{\prime}-1\right)\left(\bmod \hat{A}_{2}\right)$. Therefore $\psi\left(a_{k}\right)=k(g-1)\left(\bmod I^{2}\right)$. Hence $\psi$ is surjective.

3.3. The forms $\hat{\eta}$ and $\hat{\sigma}$. Given an F-pairing $\eta$ in $A$ and an integer $m \geq 1$, we have $\eta\left(A, I^{m}\right)=\eta\left(I, I^{m}\right) \subset I^{m-1}$ and similarly $\eta\left(I^{m}, A\right) \subset I^{m-1}$. Therefore $\eta$ induces a $\mathbb{K}$-bilinear form $\eta^{(m)}: A / I^{m} \times A / I^{m} \rightarrow A / I^{m-1}$. The forms $\left(\eta^{(m)}\right)_{m \geq 1}$ are compatible in the obvious way, and taking their projective limit we obtain a $\mathbb{K}$-bilinear pairing $\hat{\eta}: \hat{A} \times \hat{A} \rightarrow \hat{A}$. A similar construction applies to the derived form $\sigma: A \times A \rightarrow A$ of $\eta$. Lemma 2.4 and the equalities $\sigma(1, A)=\sigma(A, 1)=0$ imply that $\sigma$ induces a $\mathbb{K}$-bilinear form $A / I^{m} \times A / I^{m} \rightarrow A / I^{m-1}$ for all $m \geq 1$. 
The projective limit of these forms is a $\mathbb{K}$-bilinear pairing $\hat{\sigma}: \hat{A} \times \hat{A} \rightarrow \hat{A}$. We call $\hat{\eta}$ and $\hat{\sigma}$ the completions of $\eta$ and $\sigma$, respectively. We shall discuss the properties of $\hat{\eta}$ and $\hat{\sigma}$ in a more general context in the next subsection.

3.4. Fox pairings in $\hat{A}$. In analogy with F-pairings in $A$ we define an $F$-pairing in $\hat{A}$ to be a $\mathbb{K}$-bilinear pairing $\rho: \hat{A} \times \hat{A} \rightarrow \hat{A}$ satisfying the product formulas (2.2.1), 2.2.2) where $A$, aug, $\eta$ are replaced by $\hat{A}$, aûg, $\rho$ respectively. It follows from the product formulas that $\rho(1, \hat{A})=\rho(\hat{A}, 1)=0$. It is clear that the completion of an F-pairing in $A$ is an F-pairing in $\hat{A}$.

An F-pairing $\rho$ in $\hat{A}$ induces a bilinear form $\cdot \rho: \hat{A} \times \hat{A} \rightarrow \mathbb{K}$ by $a \cdot{ }_{\rho} b=\operatorname{aûg}(\rho(a, b))$ for $a, b \in \hat{A}$. As in Section 2.3, the pairing $: \rho \circ(\iota \times \iota): \pi \times \pi \rightarrow \mathbb{K}$ induces a bilinear form $H_{1}(\pi ; \mathbb{K}) \times H_{1}(\pi ; \mathbb{K}) \rightarrow \mathbb{K}$. The latter form is also denoted $\cdot \rho$. For any F-pairing $\eta$ in $A$ with completion $\hat{\eta}$, the form ${ }^{\eta}$ induced by $\eta$ coincides with ${ }^{\prime} \hat{\eta}$.

We now define (right) derived forms of the F-pairings in $\hat{A}$. The construction proceeds in three steps.

Step 1. The following lemma will allow us, in analogy with Section 2.1, to define for any $u \in \hat{A}$ and $v \in A$ an expression $v^{u} \in \hat{A}$.

Lemma 3.2. If $u_{1}, u_{2} \in A$ verify $u_{1} \equiv u_{2}\left(\bmod I^{m}\right)$ with $m \geq 1$, then $v^{u_{1}} \equiv$ $v^{u_{2}}\left(\bmod I^{m}\right)$ for all $v \in A$.

Proof. Since the expression $v^{u}$ is linear in $u \in A$, it suffices to show that $v^{u} \in I^{m}$ for any product $u=\left(a_{1}-1\right) \cdots\left(a_{m}-1\right)$ with $a_{1}, \ldots, a_{m} \in \pi$. This is clear for $m=1$ because $v^{a-1}=a^{-1} v a-v$ for all $a \in \pi$. For $m \geq 2$, we proceed by induction. Set $w=\left(a_{1}-1\right) \cdots\left(a_{m-1}-1\right)$. By the induction assumption, $v^{w} \in I^{m-1}$. Then

$$
v^{u}=v^{w\left(a_{m}-1\right)}=a_{m}^{-1} v^{w} a_{m}-v^{w}=a_{m}^{-1}\left(v^{w}\left(a_{m}-1\right)-\left(a_{m}-1\right) v^{w}\right) \in I^{m} .
$$

Given $u \in \hat{A}=\lim _{\longleftarrow} A / I^{m}$ and $v \in A$, we define $v^{u} \in \hat{A}$ as follows. For each $m \geq 1$, pick $u_{m} \in A$ whose projection to $A / I^{m}$ is equal to the projection of $u$ to $A / I^{m}$. By the previous lemma, $v^{u_{m}}\left(\bmod I^{m}\right)$ does not depend on the choice of $u_{m}$. Hence the sequence $v^{u_{m}}\left(\bmod I^{m}\right)$ with $m=1,2, \ldots$ represents a well-defined element $v^{u}$ of $\hat{A}$. It is clear that $v^{u}$ is linear in both $u$ and $v$. If $u=\iota\left(u^{\prime}\right)$ for some $u^{\prime} \in A$, then $v^{u}=\iota\left(v^{u^{\prime}}\right)$. The identities (2.1.1) remain true for all $u \in \hat{A}, v \in A, a \in \pi$.

Step 2. In the sequel, we treat $\hat{A}$ as an $A$-bimodule via $\iota$. Thus, given $a \in A$ and $b \in \hat{A}$, we write $a b$ for $\iota(a) b$ and $b a$ for $b \iota(a)$. Starting from an F-pairing $\rho$ in $\hat{A}$, we define a pairing $\sigma=\sigma^{\rho}: A \times A \rightarrow \hat{A}$ as follows. For any $a, b \in \pi$, set

$$
\sigma(a, b)=b a^{\rho(\iota(a), \iota(b))} \in \hat{A} .
$$

Then extend to $A \times A$ by $\mathbb{K}$-bilinearity. Clearly, $\sigma(1, A)=\sigma(A, 1)=0$ and $\operatorname{aûg}(\sigma(a, b))=\iota(a) \cdot \iota^{\prime}(b)$ for all $a, b \in A$. Further properties of $\sigma$ are summarized in the following lemma whose proof is a direct adaptation of the proofs of the parallel claims for F-pairings in $A$, see Section 2

Lemma 3.3. The form $\sigma=\sigma^{\rho}$ satisfies the following:

(i) $\sigma(a, b c)=\sigma(a, b) c+b \sigma(a, c)$ and $\sigma(a b, c)=\sigma(b a, c)$ for any $a, b, c \in A$;

(ii) $\sigma\left(A, I^{m}\right) \subset \hat{A}_{m-1}$ and $\sigma\left(I^{m}, A\right) \subset \hat{A}_{m-1}$ for any $m \geq 1$.

Step 3. Lemma 3.3 (ii) implies that for every integer $m \geq 1$, the form $\sigma$ induces a bilinear form $\sigma^{(m)}: A / I^{m} \times A / I^{m} \rightarrow \hat{A} / \hat{A}_{m-1} \simeq A / I^{m-1}$. These forms are 
compatible in the obvious way and their projective limit is a bilinear pairing $\hat{\sigma}=$ $\hat{\sigma}^{\rho}: \hat{A} \times \hat{A} \rightarrow \hat{A}$. This is the derived form of $\rho$.

The following properties of $\hat{\sigma}$ are consequences of the definitions and Lemma 3.3

- For all $m \geq 1$, we have $\hat{\sigma}\left(\hat{A}_{m}, \hat{A}\right) \subset \hat{A}_{m-1} \supset \hat{\sigma}\left(\hat{A}, \hat{A}_{m}\right)$. Applying the same argument as in the proof of Lemma 2.4 to $\sigma^{(m+n-1)}$, we easily obtain that $\hat{\sigma}\left(\hat{A}_{m}, \hat{A}_{n}\right) \subset \hat{A}_{m+n-2}$ for all $m, n \geq 1$.

- For any $a \in \hat{A}$, the map $\hat{A} \rightarrow \hat{A}, b \mapsto \hat{\sigma}(a, b)$ is a derivation of $\hat{A}$. This derivation is denoted $\hat{\sigma}(a,-)$.

- For any $a \in \hat{A}$ and $c \in \hat{\pi}$, we have $\hat{\sigma}\left(c a c^{-1},-\right)=\hat{\sigma}(a,-)$.

The definition of the derived form is compatible with completion: if $\rho=\hat{\eta}$ is the completion of an F-pairing $\eta$ in $A$, then $\sigma^{\rho}=\iota \sigma^{\eta}: A \times A \rightarrow \hat{A}$ and $\hat{\sigma}^{\rho}=\widehat{\sigma^{\eta}}$.

3.5. Filtered Fox pairings in $\hat{A}$. We call an F-pairing $\rho$ in $\hat{A}$ filtered if $\rho\left(\hat{A}_{m}, \hat{A}\right) \subset$ $\hat{A}_{m-1} \supset \rho\left(\hat{A}, \hat{A}_{m}\right)$ for all $m \geq 1$. For example, the completion of any F-pairing in $A$ is filtered.

A filtered F-pairing $\rho$ in $\hat{A}$ has several nice features which we now discuss. First of all, $\rho$ induces for every $m \geq 1$ a pairing $\rho^{(m)}: A / I^{m} \times A / I^{m} \rightarrow A / I^{m-1}$ where we use that $\hat{A} / \hat{A}_{m} \simeq A / I^{m}$. Clearly, $\rho=\varliminf_{\longleftarrow} \rho^{(m)}$. Using $\rho^{(m+n-1)}$, one easily checks that $\rho\left(\hat{A}_{m}, \hat{A}_{n}\right) \subset \hat{A}_{m+n-2}$ for all $m, n \geq 1$.

Secondly, there is an alternative description of the form ${ }^{\circ}$ in $H=H_{1}(\pi ; \mathbb{K})$. This form can be obtained by restricting $\rho^{(2)}$ to $I / I^{2} \times I / I^{2}$ and transporting along the canonical isomorphism $I / I^{2} \simeq H$. Moreover, for any $a, b \in \hat{A}$,

$$
\text { aûg } \hat{\sigma}^{\rho}(a, b)=a \cdot{ }_{\rho} b=\rho^{(2)}(p(a), p(b))
$$

where $p$ is the projection $\hat{A} \rightarrow \hat{A} / \hat{A}_{2} \simeq A / I^{2}$.

Thirdly, there is an expansion of $\hat{\sigma}^{\rho}$, viewed as a $\mathbb{K}$-linear map $\hat{A} \hat{\otimes} \hat{A} \rightarrow \hat{A}$, in terms of the Hopf algebra operations in $\hat{A}$ : the comultiplication $\hat{\Delta}: \hat{A} \rightarrow \hat{A} \hat{\otimes} \hat{A}$, the antipode $S: \hat{A} \rightarrow \hat{A}$, and the multiplication $\mu: \hat{A} \hat{\otimes} \hat{A} \rightarrow \hat{A}$.

Lemma 3.4. If $\rho$ is a filtered F-pairing in $\hat{A}$, then

$$
\hat{\sigma}^{\rho}=\mu(\mu \hat{\otimes} \mu) P_{4213}\left(\operatorname{id}_{\hat{A}} \hat{\otimes}\left(S \hat{\otimes} \operatorname{id}_{\hat{A}}\right) \hat{\Delta} \rho \hat{\otimes} \operatorname{id}_{\hat{A}}\right)(\hat{\Delta} \hat{\otimes} \hat{\Delta}): \hat{A} \hat{\otimes} \hat{A} \rightarrow \hat{A},
$$

where $P_{4213}$ is the automorphism of $\hat{A}^{\hat{\otimes} 4}$ carrying $a_{1} \hat{\otimes} a_{2} \hat{\otimes} a_{3} \hat{\otimes} a_{4}$ to $a_{4} \hat{\otimes} a_{2} \hat{\otimes} a_{1} \hat{\otimes} a_{3}$ for all $a_{1}, a_{2}, a_{3}, a_{4} \in \hat{A}$.

Proof. Both sides of (3.5.2) are $\mathbb{K}$-linear maps $\hat{A} \hat{\otimes} \hat{A} \rightarrow \hat{A}$. Both sides carry $\hat{A}_{m} \otimes \hat{A}_{n}$ to $\hat{A}_{m+n-2}$ for all $m, n \geq 1$. Indeed, each map on the right-hand side of (3.5.2) preserves the natural filtration, except for $\rho$ which may decrease the filtration degree by 2. Therefore it is enough to check (3.5.2) on the set $\{a \hat{\otimes} b: a, b \in \pi\} \subset \hat{A} \hat{\otimes} \hat{A}$ where it follows directly from the definitions.

Every F-pairing $\rho$ in $\hat{A}$ induces a filtered F-pairing $\hat{\rho}$ in $\hat{A}$ as follows. It is clear that $\rho_{0}=\rho(\iota \times \iota): A \times A \rightarrow \hat{A}$ satisfies $\rho_{0}\left(A, I^{m}\right) \subset \hat{A}_{m-1} \supset \rho_{0}\left(I^{m}, A\right)$ for all $m \geq 1$. Therefore $\rho_{0}$ induces a bilinear form $\rho_{0}^{(m)}: A / I^{m} \times A / I^{m} \rightarrow A / I^{m-1}$ for all $m \geq 1$. Then $\hat{\rho}=\lim \rho_{0}^{(m)}$ is a filtered F-pairing in $\hat{A}$. It follows from the definitions that $\cdot \rho={ }^{\circ}, \overleftarrow{\sigma^{\rho}}=\sigma^{\hat{\rho}}$, and $\hat{\sigma}^{\rho}=\hat{\sigma}^{\hat{\rho}}$. 


\section{EXPONENTIATION AND THE TWISTS}

Starting from this section we suppose that $\mathbb{Q} \subset \mathbb{K}$. Given a Fox pairing in $\hat{A}$, we use its derived form to construct automorphisms of $\hat{A}$ called the "twists."

4.1. Exponentiation. We say that a $\mathbb{K}$-linear homomorphism $f: \hat{A} \rightarrow \hat{A}$ is weakly nilpotent if for any $m \geq 0$ we have $f\left(\hat{A}_{m}\right) \subset \hat{A}_{m}$ and there is $N=N(m)>0$ such that $f^{N}(\hat{A}) \subset \hat{A}_{m}$. A weakly nilpotent homomorphism $f: \hat{A} \rightarrow \hat{A}$ has an exponent $\mathrm{e}^{f}: \hat{A} \rightarrow \hat{A}$ carrying any $a \in \hat{A}$ to

$$
\mathrm{e}^{f}(a)=\sum_{k \geq 0} \frac{f^{k}}{k !}(a)
$$

The sum on the right-hand side is well-defined because for any given $m \geq 1$, it contains only a finite number of terms that are non-zero in $\hat{A} / \hat{A}_{m} \simeq A / I^{m}$. Therefore this sum defines a $\mathbb{K}$-linear homomorphism $\left(\mathrm{e}^{f}\right)^{(m)}: A / I^{m} \rightarrow A / I^{m}$ for each $m$. These homomorphisms are compatible with respect to the projections $A / I^{m+1} \rightarrow A / I^{m}$. By definition, we have $\mathrm{e}^{f}=\varliminf_{\longleftarrow}\left(\mathrm{e}^{f}\right)^{(m)}: \hat{A} \rightarrow \hat{A}$. It follows directly from the definitions that $\mathrm{e}^{f}\left(\hat{A}_{m}\right) \subset \hat{A}_{m}$ for all $m$.

If $f: \hat{A} \rightarrow \hat{A}$ is a weakly nilpotent homomorphism, then so is $-f: \hat{A} \rightarrow \hat{A}$. The standard properties of the exponentiation give $\mathrm{e}^{f} \mathrm{e}^{-f}=\mathrm{e}^{-f} \mathrm{e}^{f}=\mathrm{id}_{\hat{A}}$. Thus, the homomorphism e $\mathrm{e}^{f}$ is invertible. The inclusions $\mathrm{e}^{f}\left(\hat{A}_{m}\right) \subset \hat{A}_{m}$ and $\mathrm{e}^{-f}\left(\hat{A}_{m}\right) \subset \hat{A}_{m}$ imply that $\mathrm{e}^{f}\left(\hat{A}_{m}\right)=\hat{A}_{m}$ for all $m$.

We call an automorphism of $\hat{A}$ filtered if it carries $\hat{A}_{m}$ onto itself for all $m$. We just showed that $\mathrm{e}^{f}$ is filtered.

If $f$ is a weakly nilpotent derivation of $\hat{A}$, then $\mathrm{e}^{f}$ is an algebra automorphism of $\hat{A}$. Indeed, for any $a, b \in \hat{A}$ and $k \geq 0$,

$$
f^{k}(a b)=\sum_{k_{1}, k_{2} \geq 0, k_{1}+k_{2}=k} \frac{k !}{k_{1} ! k_{2} !} f^{k_{1}}(a) f^{k_{2}}(b) .
$$

Substituting in the definition of $\mathrm{e}^{f}(a b)$ we obtain that $\mathrm{e}^{f}(a b)=\mathrm{e}^{f}(a) \mathrm{e}^{f}(b)$. Also $f(1)=0$ and hence $\mathrm{e}^{f}(1)=1$. Note that aûg $\circ \mathrm{e}^{f}=$ aûg. In fact, any filtered algebra automorphism of $\hat{A}$ commutes with aûg. Indeed, the induced $\mathbb{K}$-linear automorphism of $\hat{A} / \hat{A}_{1} \simeq A / I \simeq \mathbb{K}$ carries 1 to itself and has to be the identity.

4.2. The twists. Given an F-pairing $\rho$ in $\hat{A}$, it is natural to ask for which $a \in \hat{A}$ the derivation $\hat{\sigma}(a,-)=\hat{\sigma}^{\rho}(a,-)$ of $\hat{A}$ is weakly nilpotent. For example, if $a \in \hat{A}_{3}$, then $\hat{\sigma}(a,-)$ is weakly nilpotent. This follows from the inclusion $\hat{\sigma}(a,-)\left(\hat{A}_{m}\right) \subset \hat{A}_{m+1}$ for all $m \geq 0$. We exhibit now a bigger class of weakly nilpotent derivations.

Lemma 4.1. Let $a=k(c-1)^{2}+e$ where $k \in \mathbb{K}, c \in \hat{\pi} \subset \hat{A}$ with $c \cdot \rho c=0$, and $e \in \hat{A}_{3}$. Then the derivation $\hat{\sigma}(a,-)$ is weakly nilpotent.

Proof. Replacing if necessary $\rho$ by $\hat{\rho}$ we can assume that $\rho$ is filtered. Since $a \in \hat{A}_{2}$, we have $\hat{\sigma}(a,-)\left(\hat{A}_{m}\right) \subset \hat{A}_{m}$ for all $m \geq 0$. To prove that $\hat{\sigma}(a,-)$ is weakly nilpotent it is enough to show that $(\hat{\sigma}(a,-))^{m+1}\left(\hat{A}_{m}\right) \subset \hat{A}_{m+1}$ for all $m$. For $m=0$, this inclusion follows from Formula (3.5.1) because $a \in \hat{A}_{2}=\operatorname{Ker} p$. Assume now that $m \geq 1$. We have $\hat{\sigma}(a,-)=k d+d^{\prime}$ where $d=\hat{\sigma}\left((c-1)^{2},-\right)$ and $d^{\prime}=\hat{\sigma}(e,-)$. Therefore $(\hat{\sigma}(a,-))^{m+1}$ is a homogeneous polynomial in $d$ and $d^{\prime}$ of degree $m+1$. Both $d$ and $d^{\prime}$ carry $\hat{A}_{m}$ to itself and $d^{\prime}\left(\hat{A}_{m}\right) \subset \hat{A}_{m+1}$ since $e \in \hat{A}_{3}$. Hence, any 
monomial in $d$ and $d^{\prime}$ containing at least one entry of $d^{\prime}$ carries $\hat{A}_{m}$ to $\hat{A}_{m+1}$. It remains to show that $d^{m+1}\left(\hat{A}_{m}\right) \subset \hat{A}_{m+1}$.

We claim that for any $b \in \hat{\pi}$,

$$
d(b) \equiv 2(c \cdot \rho)(c-1)\left(\bmod \hat{A}_{2}\right) .
$$

To see this, pick any $s=\sum_{x \in \pi} k_{x} x \in A$ with $k_{x} \in \mathbb{K}$ so that $\rho(c, b)\left(\bmod \hat{A}_{2}\right)=$ $s\left(\bmod I^{2}\right)$. Since $c$ and $b$ are group-like, (3.5.2) gives

$$
\hat{\sigma}(c, b) \equiv \sum_{x \in \pi} k_{x} b x^{-1} c x\left(\bmod \hat{A}_{2}\right) .
$$

Note that $\rho\left(c^{2}, b\right) \equiv(1+c) \sum_{x \in \pi} k_{x} x\left(\bmod \hat{A}_{2}\right)$ and a similar computation gives

$$
\hat{\sigma}\left(c^{2}, b\right) \equiv 2 \sum_{x \in \pi} k_{x} b x^{-1} c^{2} x\left(\bmod \hat{A}_{2}\right) .
$$

Thus, we have

$$
\begin{aligned}
d(b)=\hat{\sigma}\left(c^{2}-2 c+1, b\right)=\hat{\sigma}\left(c^{2}, b\right)-2 \hat{\sigma}(c, b) & \equiv 2 b \sum_{x \in \pi} k_{x} x^{-1} c(c-1) x\left(\bmod \hat{A}_{2}\right) \\
& \equiv 2 \sum_{x \in \pi} k_{x}(c-1)\left(\bmod \hat{A}_{2}\right),
\end{aligned}
$$

and the congruence (4.2.1) follows.

The $\mathbb{K}$-module $\hat{A}_{m}$ is linearly generated by $\hat{A}_{m+1}$ and products of the form $\Pi=\left(b_{1}-1\right) \cdots\left(b_{m}-1\right)$ with $b_{1}, \ldots, b_{m} \in \iota(\pi) \subset \hat{\pi}$. As we know, $d\left(\hat{A}_{m+1}\right) \subset \hat{A}_{m+1}$, and we need only to prove that $d^{m+1}(\Pi) \in \hat{A}_{m+1}$ for all $\Pi$ as above. Applying the derivation $d$ to $\Pi$ we obtain (modulo $\hat{A}_{m+1}$ ) a sum of $m$ similar products in which one of the factors $b_{i}-1$ is transformed into $d\left(b_{i}-1\right)=d\left(b_{i}\right) \equiv 2\left(c \cdot b_{\rho} b_{i}\right)(c-1)$ while the other factors are preserved. Applying $d$ recursively $m+1$ times to $\Pi$, we necessarily have to transform one of the factors twice. This gives 0 because $c \cdot{ }_{\rho} c=0$. Hence $d^{m+1}\left(\hat{A}_{m}\right) \subset \hat{A}_{m+1}$.

Lemma 4.1 implies that every $a \in \hat{A}$ as in this lemma gives rise to a filtered algebra automorphism $\mathrm{e}^{\hat{\sigma}(a,-)}$ of $\hat{A}$. In particular, for $k \in \mathbb{K}$ and $\alpha \in \hat{\pi}$ such that $\alpha \cdot \rho \alpha=0$, we set

$$
t_{k, \alpha}=\mathrm{e}^{\hat{\sigma}\left(k \log ^{2}(\alpha),-\right)}=\mathrm{e}^{k \hat{\sigma}\left(\log ^{2}(\alpha),-\right)}: \hat{A} \rightarrow \hat{A} .
$$

Here

$$
\log (\alpha)=\log (1+(\alpha-1))=(\alpha-1)-\frac{(\alpha-1)^{2}}{2}+\frac{(\alpha-1)^{3}}{3}-\cdots
$$

is a well-defined element of $\hat{A}$ and $\log ^{2}(\alpha)=(\log (\alpha))^{2}$. Note that $\log ^{2}(\alpha) \epsilon$ $(\alpha-1)^{2}+\hat{A}_{3}$. By Lemma 4.1, the derivation $\hat{\sigma}\left(k \log ^{2}(\alpha),-\right)$ is weakly nilpotent. Therefore $t_{k, \alpha}$ is a well-defined filtered algebra automorphism of $\hat{A}$. We call $t_{k, \alpha}$ the twist of $\hat{A}$ determined by $\rho, k$, and $\alpha$.

4.3. Properties of the twists. We state several properties of the twists. Fix an F-pairing $\rho$ in $\hat{A}$ and $\alpha \in \hat{\pi}$ such that $\alpha{ }^{\circ} \alpha=0$.

- We have $t_{k, c \alpha c^{-1}}=t_{k, \alpha}$ for all $k \in \mathbb{K}$ and $c \in \hat{\pi}$. This follows from the conjugation invariance of $\hat{\sigma}$.

- We have $t_{k+l, \alpha}=t_{k, \alpha} t_{l, \alpha}$ for all $k, l \in \mathbb{K}$. This follows from the properties of the exponentiation. In particular, $t_{k, \alpha}$ commutes with $t_{l, \alpha}$ for all $k, l \in \mathbb{K}$. 
- We have $t_{m k, \alpha}=\left(t_{k, \alpha}\right)^{m}$ for all $m \in \mathbb{Z}$ and $k \in \mathbb{K}$. In particular, $t_{0, \alpha}=\mathrm{id}_{\hat{A}}$ and $t_{-k, \alpha}=t_{k, \alpha}^{-1}$. This follows from the previous property.

- We have $t_{k, \alpha^{m}}=t_{m^{2} k, \alpha}=\left(t_{k, \alpha}\right)^{m^{2}}$ for all $k \in \mathbb{K}$ and $m \in \mathbb{Z}$. This follows from the equality $\log \left(\alpha^{m}\right)=m \log (\alpha)$. In particular, $t_{k, \alpha^{-1}}=t_{k, \alpha}$ for all $k \in \mathbb{K}$.

- For all $m \geq 2$, the automorphism $t_{k, \alpha}^{(m)}$ of $\hat{A} / \hat{A}_{m} \simeq A / I^{m}$ induced by $t_{k, \alpha}$ depends only on $k$ and the image of $\alpha$ in $\hat{\pi} / \hat{\pi}_{(m)}$. This follows from the following claim: if $\beta \in \hat{\pi}$ is such that $\alpha-\beta \in \hat{A}_{m}$, then $t_{k, \alpha}^{(m)}=t_{k, \beta}^{(m)}$. Indeed,

$$
\log (\alpha)-\log (\beta) \in \hat{A}_{m} \quad \text { and so } \quad \log ^{2}(\alpha)-\log ^{2}(\beta) \in \hat{A}_{m+1} \text {. }
$$

Therefore $\hat{\sigma}\left(\log ^{2}(\alpha),-\right) \equiv \hat{\sigma}\left(\log ^{2}(\beta),-\right)\left(\bmod \hat{A}_{m}\right)$. Hence the claim.

- We have $t_{k, \alpha}(\beta)=\beta$ for all $k \in \mathbb{K}$ and all $\beta \in \hat{\pi}$ such that $\rho(\alpha, \beta)=0$. Indeed, if $\rho(\alpha, \beta)=0$, then $\rho\left(\alpha^{m}, \beta\right)=0$ for all $m \geq 0$. Since $\alpha^{m}$ and $\beta$ are group-like, Formula (3.5.2) gives $\hat{\sigma}\left(\alpha^{m}, \beta\right)=0$ for all $m$. Hence $\hat{\sigma}\left(\log ^{2}(\alpha),-\right)(\beta)=0$.

Any filtered automorphism $\varphi$ of $\hat{A}$ induces a $\mathbb{K}$-linear automorphism $\varphi_{*}$ of $H=$ $H_{1}(\pi ; \mathbb{K}) \simeq I / I^{2} \simeq \hat{A}_{1} / \hat{A}_{2}$. The computations in the proof of Lemma 4.1 imply that, for all $h \in H$,

$$
\left(t_{k, \alpha}\right)_{*}(h)=h+2 k([\alpha] \cdot \rho h)[\alpha]
$$

where $[\alpha] \in H$ is the image of $\alpha$ under the projection $\hat{\pi} \rightarrow \hat{\pi} / \hat{\pi}_{(2)} \simeq H$. Thus, the twist $t_{k, \alpha}$ induces a transvection of $H$.

All the constructions above are natural with respect to isomorphisms of groups such that the induced isomorphisms of the completed group algebras preserve the F-pairings. Applying this principle to automorphisms of $\hat{A}$ we obtain the following lemma. Here and in the sequel we say that a map $\varphi$ from a set $L$ to itself preserves a pairing $\rho: L \times L \rightarrow L$ if $\rho(\varphi(a), \varphi(b))=\varphi(\rho(a, b))$ for all $a, b \in L$.

Lemma 4.2. Let $\varphi$ be a $\rho$-preserving automorphism of $\hat{A}$ induced by an automorphism of $\pi$. Then for all $k \in \mathbb{K}$ and $\alpha \in \hat{\pi}$ such that $\alpha{ }_{\rho} \alpha=0$, the following diagram commutes:

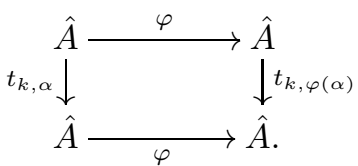

4.4. Remark. If $\mathbb{K}=\mathbb{R}$ and the group $\pi$ is finitely generated, then we can define $t_{k, \alpha}$ for all $\alpha \in \hat{\pi}$ (i.e., without the assumption $\alpha{ }_{\rho} \alpha=0$ ). We proceed as follows. For each $m \geq 1$, let $d_{m}$ be the derivation of the algebra $\hat{A} / \hat{A}_{m} \simeq A / I^{m}$ induced by $d=\hat{\sigma}\left(k \log ^{2}(\alpha),-\right): \hat{A} \rightarrow \hat{A}$. Since $\mathbb{K}=\mathbb{R}$ and $\pi$ is finitely generated, $A / I^{m}$ is a finite dimensional real vector space. Then the map $d_{m}$ has an exponential $\mathrm{e}^{d_{m}}: A / I^{m} \rightarrow A / I^{m}$, and the projective limit $t_{k, \alpha}=\lim _{\longleftarrow} \mathrm{e}^{d_{m}}$ is a well-defined automorphism of $\hat{A}$. It generalizes the twist defined above in the case $\alpha \cdot{ }_{\rho} \alpha=0$ and has similar properties. Note that $t_{k, \alpha}$ is differentiable as a function of $k$ and its first derivative is equal to $\hat{\sigma}\left(\log ^{2}(\alpha),-\right) t_{k, \alpha}$. We do not dwell on this construction because, in our main application to surfaces, the form $\cdot \rho$ is skew-symmetric and the condition $\alpha \cdot{ }_{\rho} \alpha=0$ is met for all $\alpha \in \hat{\pi}$.

\section{Twists as Hopf Algebra Automorphisms}

We now show that the twists, which we defined in the previous section, preserve the complete Hopf algebra structure of $\hat{A}$. 
5.1. H-automorphisms of $\hat{A}$. By an H-automorphism of $\hat{A}$ we mean a $\mathbb{K}$-linear filtered algebra isomorphism $\varphi: \hat{A} \rightarrow \hat{A}$ preserving comultiplication. Thus, $\varphi$ is an algebra isomorphism, $\varphi\left(\hat{A}_{m}\right)=\hat{A}_{m}$ for all $m \geq 1$, and $\hat{\Delta} \varphi=(\varphi \hat{\otimes} \varphi) \hat{\Delta}$. The group of H-automorphisms of $\hat{A}$ is $\operatorname{denoted} \operatorname{Aut}(\hat{A})$. Note that any H-automorphism of $\hat{A}$ commutes with the counit aûg because all filtered algebra automorphisms of $\hat{A}$ do so, see Section 4.1 Any H-automorphism $\varphi$ of $\hat{A}$ commutes with the antipode $S$ : since $\left.S\right|_{\hat{\pi}}$ is the group inversion and $\left.\varphi\right|_{\hat{\pi}}$ is an automorphism of $\hat{\pi}$ we have $\left.S \varphi\right|_{\hat{\pi}}=\left.\varphi S\right|_{\hat{\pi}}$; since $\hat{\pi}$ generates $\hat{A} / \hat{A}_{m}$ as a $\mathbb{K}$-module for all $m$, we have $S \varphi=\varphi S$.

An automorphism of the group $\hat{\pi} \subset \hat{A}$ is said to be filtered if it carries $\hat{\pi}_{(m)}=$ $\hat{\pi} \cap\left(1+\hat{A}_{m}\right)$ onto itself for all $m \geq 1$. Denote the group of filtered automorphisms of $\hat{\pi}$ by $\operatorname{Aut}(\hat{\pi})$. Each H-automorphism of $\hat{A}$ restricts to a filtered automorphism of $\hat{\pi}$. Since $\hat{\pi}$ generates the $\mathbb{K}$-module $\hat{A} / \hat{A}_{m}$ for all $m$, the restriction to $\hat{\pi}$ defines an injective group homomorphism

$$
\operatorname{Aut}(\hat{A}) \longrightarrow \operatorname{Aut}(\hat{\pi}) .
$$

For $\mathbb{K}=\mathbb{Q}$, this homomorphism is an isomorphism: see $\mathrm{Qu}$, Theorem 3.3.

Theorem 5.1. Let $\rho$ be an F-pairing in $\hat{A}$. For any $k \in \mathbb{K}$ and any $\alpha \in \hat{\pi}$ such that $\alpha \cdot{ }_{\rho} \alpha=0$, the twist $t_{k, \alpha}$ is an H-automorphism of $\hat{A}$. Consequently, $t_{k, \alpha}(\hat{\pi})=\hat{\pi}$.

The restriction of $t_{k, \alpha}$ to $\hat{\pi}$ is a filtered group automorphism of $\hat{\pi}$ also denoted $t_{k, \alpha}$ and called the twist. As we saw in Section 4.3, the induced automorphism $\left(t_{k, \alpha}\right)_{*}$ of $H=H_{1}(\pi ; \mathbb{K}) \simeq \hat{\pi} / \hat{\pi}_{(2)}$ is the transvection carrying any $h \in H$ to $\left(t_{k, \alpha}\right)_{*}(h)=$ $h+2 k([\alpha] \cdot \rho)[\alpha]$. Thus we view $t_{k, \alpha}: \hat{\pi} \rightarrow \hat{\pi}$ as a non-abelian transvection. The injectivity of (5.1.1) shows that we loose no information restricting the twists from $\hat{A}$ to $\hat{\pi}$. Generally speaking, the homomorphism $\left(t_{k, \alpha}\right)_{*}: H \rightarrow H$ does not preserve the form $\cdot \rho$ in $H$. It does preserve $\bullet_{\rho}$ if $[\alpha] \cdot \rho+h \bullet_{\rho}[\alpha]=0$ for all $h \in H$.

5.2. Proof of Theorem 5.1, Our first step is to define $v^{u}$ for all $u, v \in \hat{A}$.

Lemma 5.2. If $v_{1}, v_{2} \in A$ verify $v_{1} \equiv v_{2}\left(\bmod I^{m}\right)$ with $m \geq 1$, then $v_{1}^{u} \equiv$ $v_{2}^{u}\left(\bmod I^{m}\right)$ for all $u \in A$.

Proof. This lemma follows from the linearity of $v^{u}$ with respect to $v \in A$ and the fact that $I^{m}$ is a two-sided ideal of $A$.

Given $u, v \in \hat{A}$, we define $v^{u} \in \hat{A}$ as follows. For each $m \geq 1$, pick $u_{m}, v_{m} \in A$ such that their projections to $A / I^{m}$ are equal to the projections of $u, v$ to $A / I^{m}$, respectively. By Lemmas 3.2 and $5.2,\left(v_{m}\right)^{u_{m}}\left(\bmod I^{m}\right)$ does not depend on the choice of $u_{m}$ and $v_{m}$. Hence the sequence $\left(v_{m}\right)^{u_{m}}\left(\bmod I^{m}\right)$ with $m=1,2, \ldots$ represents a well-defined element $v^{u}$ of $\hat{A}$. Clearly, $v^{u}$ is linear in both $u$ and $v$. If $v=\iota\left(v^{\prime}\right)$ for some $v^{\prime} \in A$, then $v^{u}=\left(v^{\prime}\right)^{u}$ with the notation of Section 3.4.

Note that, given a formal power series $u(z) \in \mathbb{K}\langle\langle z-1\rangle\rangle$, we can substitute any $a \in 1+\hat{A}_{1}$ for $z$ and obtain thus a well-defined element $u(a)$ of $\hat{A}$.

Lemma 5.3. If $\rho$ is filtered, then for any $a, b \in \hat{\pi}$ and $k \in \mathbb{K}$,

$$
\hat{\sigma}\left(k \log ^{2}(a), b\right)=2 k b(\log (a))^{\rho(a, b)} \in \hat{A} .
$$

Proof. We shall prove the following more general statement: For any formal power series $u(z) \in \mathbb{K}\langle\langle z-1\rangle\rangle$,

$$
\hat{\sigma}(u(a), b)=b\left(a u^{\prime}(a)\right)^{\rho(a, b)} \in \hat{A} .
$$


Here $u^{\prime}(z) \in \mathbb{K}\langle\langle z-1\rangle\rangle$ is the formal derivative of $u(z)$. For $u(z)=k \log ^{2}(z)$, we have $z u^{\prime}(z)=2 k \log (z)$ and (5.2.2) specializes to (5.2.1).

It is enough to prove (5.2.2) modulo $\hat{A}_{m}$ for each $m \geq 1$. Fix $m$ and expand $\rho^{(m+1)}(a, b)=\sum_{x \in \pi} k_{x} x\left(\bmod I^{m}\right)$ where $k_{x} \in \mathbb{K}$ and $k_{x}=0$ except for a finite set of $x \in \pi$. For each $n \geq 1$,

$$
\rho^{(m+1)}\left(a^{n}, b\right)=\sum_{i=0}^{n-1} a^{i} \rho^{(m+1)}(a, b)=\sum_{i=0}^{n-1} \sum_{x \in \pi} k_{x} a^{i} x\left(\bmod I^{m}\right) .
$$

Formula (3.5.2) implies that modulo $\hat{A}_{m}$

$$
\hat{\sigma}\left(a^{n}, b\right) \equiv \sum_{i=0}^{n-1} \sum_{x \in \pi} k_{x} b\left(a^{i} x\right)^{-1} a^{n} a^{i} x=\sum_{x \in \pi} n k_{x} b x^{-1} a^{n} x .
$$

Then, for all $N \geq 1$, we have the following equalities and congruences modulo $\hat{A}_{m}$ :

$$
\begin{aligned}
\hat{\sigma}\left((a-1)^{N}, b\right) & =\sum_{n=0}^{N}(-1)^{N-n}\left(\begin{array}{c}
N \\
n
\end{array}\right) \hat{\sigma}\left(a^{n}, b\right) \\
& \equiv \sum_{x \in \pi} k_{x}\left(\sum_{n=1}^{N}(-1)^{N-n}\left(\begin{array}{c}
N \\
n
\end{array}\right) n b x^{-1} a^{n} x\right) \\
& =b \sum_{x \in \pi} k_{x} x^{-1}\left(N \sum_{n=1}^{N}(-1)^{N-n} \frac{(N-1) !}{(n-1) !(N-n) !} a^{n}\right) x \\
& =b \sum_{x \in \pi} k_{x} x^{-1} N a(a-1)^{N-1} x \\
& =b\left(N a(a-1)^{N-1}\right)^{\rho^{(m+1)}(a, b)} \equiv b\left(N a(a-1)^{N-1}\right)^{\rho(a, b)} .
\end{aligned}
$$

Hence $\hat{\sigma}(u(a), b) \equiv b\left(a u^{\prime}(a)\right)^{\rho(a, b)}\left(\bmod \hat{A}_{m}\right)$. This implies (5.2.2).

Lemma 5.4. Let $d: \hat{A} \rightarrow \hat{A}$ be a coderivation in the sense that $d$ is $\mathbb{K}$-linear and

$$
\hat{\Delta} d=(d \hat{\otimes} \mathrm{id}+\mathrm{id} \hat{\otimes} d) \hat{\Delta}: \hat{A} \rightarrow \hat{A} \hat{\otimes} \hat{A} .
$$

If $d$ is weakly nilpotent, then $\mathrm{e}^{d}: \hat{A} \rightarrow \hat{A}$ preserves the comultiplication in $\hat{A}$.

Proof. We have

$$
\begin{aligned}
\hat{\Delta} \mathrm{e}^{d}=\sum_{r \geq 0} \frac{1}{r !} \hat{\Delta} d^{r} & =\sum_{r \geq 0} \frac{1}{r !}(d \hat{\otimes} \mathrm{id}+\mathrm{id} \hat{\otimes} d)^{r} \hat{\Delta} \\
& =\sum_{r \geq 0} \frac{1}{r !} \sum_{i=0}^{r}\left(\begin{array}{l}
r \\
i
\end{array}\right)(d \hat{\otimes} \mathrm{id})^{i}(\mathrm{id} \hat{\otimes} d)^{r-i} \hat{\Delta} \\
& =\sum_{i \geq 0} \sum_{j \geq 0} \frac{1}{i ! j !}\left(d^{i} \hat{\otimes} d^{j}\right) \hat{\Delta}=\left(\mathrm{e}^{d} \hat{\otimes} \mathrm{e}^{d}\right) \hat{\Delta}
\end{aligned}
$$

Lemma 5.5. $d=\hat{\sigma}\left(k \log ^{2}(a),-\right): \hat{A} \rightarrow \hat{A}$ is a coderivation for all $a \in \hat{\pi}, k \in \mathbb{K}$. 
Proof. Replacing if necessary $\rho$ by $\hat{\rho}$ we can assume that $\rho$ is filtered. Both sides of (5.2.3) carry the canonical filtration of $\hat{A}$ into the canonical filtration of $\hat{A} \hat{\otimes} \hat{A}$. Therefore it suffices to prove the equality of the induced homomorphisms $\hat{A} / \hat{A}_{m} \rightarrow \hat{A} \hat{\otimes} \hat{A} /(\hat{A} \hat{\otimes} \hat{A})_{m}$ for all $m$. Pick any $b \in \hat{\pi}$ and expand $\rho^{(m+1)}(a, b)=$ $\sum_{x \in \pi} k_{x} x\left(\bmod I^{m}\right)$ as in the proof of the previous lemma. Formula (5.2.1) gives the following congruence modulo $\hat{A}_{m}$ :

$$
d(b) \equiv 2 k \sum_{x \in \pi} k_{x} b x^{-1} \log (a) x=2 k \sum_{x \in \pi} k_{x} b \log \left(x^{-1} a x\right) .
$$

Therefore we have the following equalities and congruences modulo $(\hat{A} \hat{\otimes} \hat{A})_{m}$ :

$$
\begin{aligned}
\hat{\Delta}(d(b)) & \equiv 2 k \sum_{x \in \pi} k_{x} \hat{\Delta}\left(b \log \left(x^{-1} a x\right)\right) \\
& =2 k \sum_{x \in \pi} k_{x} \hat{\Delta}(b) \hat{\Delta}\left(\log \left(x^{-1} a x\right)\right) \\
& =2 k \sum_{x \in \pi} k_{x}(b \hat{\otimes} b)\left(\log \left(x^{-1} a x\right) \hat{\otimes} 1+1 \hat{\otimes} \log \left(x^{-1} a x\right)\right) \\
& =2 k \sum_{x \in \pi} k_{x}\left(b \log \left(x^{-1} a x\right) \hat{\otimes} b+b \hat{\otimes} b \log \left(x^{-1} a x\right)\right) \\
& \equiv d(b) \hat{\otimes} b+b \hat{\otimes} d(b) .
\end{aligned}
$$

Here we used that $\log \left(x^{-1} a x\right)$ is primitive by (3.2.1). It remains to observe that $\hat{\pi} \supset \iota(\pi)$ and the set $\iota(\pi)$ generates $\hat{A} / \hat{A}_{m} \simeq A / I^{m}$ as a $\mathbb{K}$-module.

We can now finish the proof of Theorem 5.1. We need only to prove that $t_{k, \alpha}$ preserves comultiplication. This is a direct consequence of the previous two lemmas.

5.3. Remark. Under certain assumptions, the definitions of the Fox pairings and the twists can be generalized to Hopf algebras other than $A=\mathbb{K}[\pi]$. We plan to discuss this generalization elsewhere.

\section{NON-DEGENERATE FoX PAIRINGS}

In this section we study a useful class of non-degenerate Fox pairings.

6.1. Non-degenerate F-pairings. We call an F-pairing $\rho$ in $\hat{A}$ non-degenerate if $\rho$ is filtered, the $\mathbb{K}$-module $H=H_{1}(\pi ; \mathbb{K})$ is free of finite rank, and the matrix of the form ${ }^{\circ}: H \times H \rightarrow \mathbb{K}$ with respect to a basis of $H$ is invertible over $\mathbb{K}$. Note that the second condition holds if and only if the first Betti number of $\pi$ is finite. This follows from the equality $H=\mathbb{K} \otimes_{\mathbb{Z}} H_{1}(\pi ; \mathbb{Q})$ which holds because we have assumed that $\mathbb{K} \supset \mathbb{Q}$. We shall need the following lemma.

Lemma 6.1. Let $B$ be an $n \times n$ matrix with entries in $\hat{A}$ where $n \geq 1$. If the $n \times n$ matrix obtained from $B$ by termwise application of aûg $: \hat{A} \rightarrow \mathbb{K}$ is invertible over $\mathbb{K}$, then $B$ is invertible over $\hat{A}$.

Proof. Consider an arbitrary $\mathbb{K}$-algebra $R$ with filtration $R=R_{0} \supset R_{1} \supset R_{2} \supset \cdots$ such that $R_{i}$ is a submodule of $R$ for every $i \geq 0, R_{i} R_{j} \subset R_{i+j}$ for any $i, j \geq 0$, and the canonical map $R \rightarrow \lim R / R_{i}$ is an isomorphism. We claim that if $r, r^{\prime}$ are elements of $R$ such that $r^{\prime}-r \in R_{1}$ and $r^{\prime}$ is invertible in $R$, then $r$ is invertible in $R$. Indeed, replacing $r$ and $r^{\prime}$ by $r\left(r^{\prime}\right)^{-1}$ and 1 , respectively, we can assume that 
$r^{\prime}=1$. It is clear that $(1-r)^{i} \in R_{i}$ for all $i \geq 0$. Then $s=\sum_{i \geq 0}(1-r)^{i}$ is a well-defined element of $R$ such that $s(1-r)=s-1=(1-r) s$. Then $r s=s r=1$.

Let $R$ be the algebra of $n \times n$ matrices over $\hat{A}$ and, for any $i \geq 0$, let $R_{i} \subset R$ consist of matrices with all entries in $\hat{A}_{i}$. For $B \in R$, the matrix $B^{\prime}=\operatorname{aûg}(B)$ over $\mathbb{K}$ can be regarded as an element of $R$. Clearly, $B^{\prime}-B \in R_{1}$. Thus, the lemma follows from the claim above.

We explain now that a non-degenerate F-pairing in $\hat{A}$ is fully determined by a single element of $\hat{A}$.

Lemma 6.2. For a non-degenerate F-pairing $\rho$ in $\hat{A}$ there is a unique $\nabla_{\rho} \in \hat{A}_{1}$ such that $\rho\left(a, \nabla_{\rho}\right)=a-\operatorname{aûg}(a)$ for all $a \in \hat{A}$. The map $\rho \mapsto \nabla_{\rho}$ from the set of non-degenerate F-pairings in $\hat{A}$ to $\hat{A}_{1}$ is injective.

Proof. Pick elements $g_{1}, \ldots, g_{n}$ of $\pi$ such that the homology classes $\left[g_{1}\right], \ldots,\left[g_{n}\right] \in H$ form a basis of the $\mathbb{K}$-module $H$. We claim that for each $m \geq 1$, the set $\left(g_{r}\right)_{r}$ generates $A / I^{m}$ as an algebra. Indeed, consider a free group $F$ of rank $n$ and a group homomorphism $f: F \rightarrow \pi$ carrying a basis of $F$ into $g_{1}, \ldots, g_{n}$. Let $J$ be the fundamental ideal of $\mathbb{K}[F]$. Consider the commutative diagram

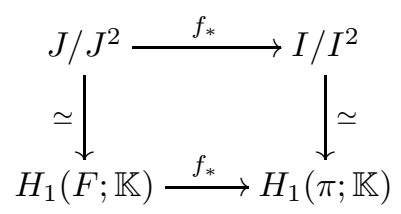

where the vertical arrows are the canonical isomorphisms and the horizontal arrows are induced by $f$. Since the bottom horizontal arrow is surjective, so is the top horizontal arrow. Multiplication in $I$ defines a surjection $\left(I / I^{2}\right)^{\otimes N} \rightarrow I^{N} / I^{N+1}$ for all $N \geq 1$, and similarly for $J$. This implies that the homomorphism $f_{*}$ : $\mathbb{K}[F] / J^{m} \rightarrow A / I^{m}$ is surjective. Hence, $\left(g_{r}\right)_{r}$ generates $A / I^{m}$ as an algebra.

To simplify the formulas, we shall use the same symbols $g_{1}, \ldots, g_{n}$ for the elements $\iota\left(g_{1}\right), \ldots, \iota\left(g_{n}\right)$ of $\hat{A}$. For $r, s=1, \ldots, n$, set $b_{r, s}=\rho\left(g_{r}, g_{s}\right) \in \hat{A}$. A termwise application of aûg to the matrix $B=\left(b_{r, s}\right)_{r, s}$ yields the invertible matrix $\left(g_{r}{ }^{\circ} g_{s}\right)_{r, s}$ over $\mathbb{K}$. By Lemma 6.1 $B$ is invertible over $\hat{A}$. Let $B^{-1}=\left(c_{r, s}\right)_{r, s}$ where $c_{r, s} \in \hat{A}$. Set

$$
\nabla=\sum_{r, s=1}^{n}\left(g_{r}-1\right) c_{r, s}\left(g_{s}-1\right) \in \hat{A}_{2} \subset \hat{A}_{1}
$$

We claim that $\nabla_{\rho}=\nabla$ satisfies the conditions of the lemma. For any $i=1, \ldots, n$,

$$
\begin{aligned}
\rho\left(g_{i}-1, \nabla\right) & =\sum_{r, s=1}^{n} \rho\left(g_{i}-1,\left(g_{r}-1\right) c_{r, s}\left(g_{s}-1\right)\right) \\
& =\sum_{r, s=1}^{n} \rho\left(g_{i}-1, g_{r}-1\right) c_{r, s}\left(g_{s}-1\right) \\
& =\sum_{r, s=1}^{n} b_{i, r} c_{r, s}\left(g_{s}-1\right)=\sum_{s=1}^{n} \delta_{i}^{s}\left(g_{s}-1\right)=g_{i}-1
\end{aligned}
$$

where $\delta_{i}^{s}$ is the Kronecker delta. Since the restriction of $\rho$ to $\hat{A}_{1} \times \hat{A}$ is $\hat{A}$-linear with respect to the first variable, $\rho(a, \nabla)=a$ for all $a \in \sum_{i} \hat{A}\left(g_{i}-1\right) \subset \hat{A}$. Therefore 
$\rho^{(m)}(a, \nabla)=a$ for all $a$ in the left ideal of the algebra $\hat{A} / \hat{A}_{m} \simeq A / I^{m}$ generated by $\left(g_{i}-1\right)_{i}$. Clearly, $\rho^{(m)}(1, \nabla)=0$. These computations and the claim established at the beginning of the proof imply that $\rho^{(m)}(a, \nabla)=a-\operatorname{aung}(a)$ for all $a \in A / I^{m}$ and all $m \geq 1$. Since $\rho=\lim \rho^{(m)}$, we have $\rho(a, \nabla)=a-\operatorname{aukg}(a)$ for all $a \in \hat{A}$.

If there are two $\nabla_{\rho}$ 's satisfying the conditions of the lemma, then their difference, $\delta \in \hat{A}_{1}$, satisfies $\rho(\hat{A}, \delta)=0$. Projecting $\delta$ to $\hat{A} / \hat{A}_{m} \simeq A / I^{m}$, we can expand $\delta=\sum_{s=1}^{n}\left(g_{s}-1\right) \delta_{s}$ where $\delta_{s} \in A / I^{m-1}$ for all $s$. Then for all $r=1, \ldots, n$,

$$
0=\rho^{(m)}\left(g_{r}, \delta\right)=\sum_{s=1}^{n} b_{r, s} \delta_{s}
$$

Since the matrix $B$ is invertible, we conclude that $\delta_{s}=0$ for all $s$. Thus, $\delta$ projects to $0 \in A / I^{m}$ for all $m$. Hence $\delta=0$.

To prove the second claim of the lemma, observe that for an arbitrary expansion (6.1.2) of $\nabla=\nabla_{\rho}$ with $c_{r, s} \in \hat{A}$, the matrices $C=\left(c_{r, s}\right)$ and $B=\left(\rho\left(g_{r}, g_{s}\right)\right)$ are mutually inverse. Indeed, for any $p, q=1, \ldots, n$,

$$
\begin{aligned}
\rho\left(g_{p}, g_{q}\right)=\rho\left(g_{p}-1, g_{q}\right)=\rho\left(\rho\left(g_{p}, \nabla\right), g_{q}\right) & =\sum_{r, s} \rho\left(\rho\left(g_{p}, g_{r}\right) c_{r, s}\left(g_{s}-1\right), g_{q}\right) \\
& =\sum_{r, s} \rho\left(g_{p}, g_{r}\right) c_{r, s} \rho\left(g_{s}, g_{q}\right) .
\end{aligned}
$$

Thus, $B=B C B$. Since $B$ is invertible, $B=C^{-1}$.

Let now $\rho_{1}$ and $\rho_{2}$ be two non-degenerate F-pairings in $\hat{A}$ such that $\nabla_{\rho_{1}}=\nabla_{\rho_{2}}$. By the above, $\rho_{1}\left(g_{r}, g_{s}\right)=\rho_{2}\left(g_{r}, g_{s}\right)$ for all $r, s$. Since $\left(g_{r}\right)_{r}$ generates $A / I^{m}$ as an algebra for all $m \geq 1$, any $u, v \in A / I^{m}$ can be expanded as

$$
u=\operatorname{aug}(u)+\sum_{r=1}^{n} u_{r}\left(g_{r}-1\right) \quad \text { and } \quad v=\operatorname{aug}(v)+\sum_{s=1}^{n}\left(g_{s}-1\right) v_{s}
$$

with $u_{r}, v_{s} \in A / I^{m-1}$. Therefore we have the following equalities in $A / I^{m-1}$ :

$$
\rho_{1}^{(m)}(u, v)=\sum_{r, s} u_{r} \rho_{1}^{(m)}\left(g_{r}, g_{s}\right) v_{s}=\sum_{r, s} u_{r} \rho_{2}^{(m)}\left(g_{r}, g_{s}\right) v_{s}=\rho_{2}^{(m)}(u, v) .
$$

Thus, $\rho_{1}^{(m)}=\rho_{2}^{(m)}$ for all $m \geq 1$. Hence $\rho_{1}=\lim _{\longleftarrow} \rho_{1}^{(m)}=\lim _{\longleftarrow} \rho_{2}^{(m)}=\rho_{2}$.

Lemma 6.3. Let $\rho$ be a non-degenerate F-pairing in $\hat{A}$. A filtered algebra automorphism $\varphi$ of $\hat{A}$ preserves $\rho$ if and only if $\varphi\left(\nabla_{\rho}\right)=\nabla_{\rho}$.

Proof. If $\varphi$ preserves $\rho$, then for any $a \in \hat{A}$,

$$
\begin{aligned}
\rho\left(a, \varphi\left(\nabla_{\rho}\right)\right) & =\rho\left(\varphi\left(\varphi^{-1}(a)\right), \varphi\left(\nabla_{\rho}\right)\right) \\
& =\varphi\left(\rho\left(\varphi^{-1}(a), \nabla_{\rho}\right)\right) \\
& =\varphi\left(\varphi^{-1}(a)-\operatorname{aûg}\left(\varphi^{-1}(a)\right)\right)=a-\operatorname{aûg}(a)
\end{aligned}
$$

where we use the fact that any filtered algebra automorphism of $\hat{A}$ commutes with aûg. By the uniqueness in Lemma 6.2, we have $\varphi\left(\nabla_{\rho}\right)=\nabla_{\rho}$.

Suppose conversely that $\varphi\left(\nabla_{\rho}\right)=\nabla_{\rho}$ and define a bilinear form $\rho^{\prime}: \hat{A} \times \hat{A} \rightarrow \hat{A}$ by $\rho^{\prime}(a, b)=\varphi^{-1}(\rho(\varphi(a), \varphi(b)))$ for $a, b \in \hat{A}$. The assumptions on $\varphi$ and $\rho$ imply that $\rho^{\prime}$ is a filtered F-pairing in $\hat{A}$. It is non-degenerate. Indeed, the form $\cdot \rho^{\prime}$ in $H_{1}(\pi ; \mathbb{K}) \simeq I / I^{2} \simeq \hat{A}_{1} / \hat{A}_{2}$ is obtained from $: \rho$ via the endomorphism of $\hat{A}_{1} / \hat{A}_{2}$ 
induced by $\varphi$. Since $\varphi$ is filtered, this endomorphism is an automorphism, and the non-degeneracy of $\rho$ implies the non-degeneracy of $\rho^{\prime}$. For any $a \in \hat{A}$,

$$
\begin{aligned}
\rho^{\prime}\left(a, \nabla_{\rho}\right)=\varphi^{-1}\left(\rho\left(\varphi(a), \varphi\left(\nabla_{\rho}\right)\right)\right) & =\varphi^{-1}\left(\rho\left(\varphi(a), \nabla_{\rho}\right)\right) \\
& =\varphi^{-1}(\varphi(a)-\operatorname{aûg}(\varphi(a)))=a-\operatorname{aûg}(a) .
\end{aligned}
$$

Thus, $\nabla_{\rho}=\nabla_{\rho^{\prime}}$. Now, Lemma 6.2 implies that $\rho=\rho^{\prime}$ which means that the automorphism $\varphi$ preserves $\rho$.

Theorem 6.4. Let $\rho$ be a non-degenerate F-pairing in $\hat{A}$ such that $\nabla_{\rho}=\nu-1$ for some $\nu \in \hat{\pi}$. Then for all $k \in \mathbb{K}$ and $\alpha \in \hat{\pi}$ with $\alpha{ }_{\rho} \alpha=0$, the twist $t_{k, \alpha}$ of $\hat{A}$ preserves both $\rho$ and $\nabla_{\rho}$.

Proof. By Lemma 6.3, it suffices to prove that $t_{k, \alpha}$ preserves $\nabla=\nabla_{\rho}$. We shall prove a more general statement: for all $a \in \hat{A}$ as in Lemma 4.1 the filtered algebra automorphism $\mathrm{e}^{\hat{\sigma}(a,-)}$ of $\hat{A}$ preserves $\nabla$. For any $g \in \hat{\pi}$, we have $\rho(g, \nu)=\rho(g, \nabla)=$ $g-1$. Formula (3.5.2) gives $\hat{\sigma}^{\rho}(g, \nu)=\nu\left(g^{-1} g g-g\right)=0$. Since $\hat{\pi}$ generates $\hat{A} / \hat{A}_{m} \simeq A / I^{m}$ as a $\mathbb{K}$-module for all $m$, we deduce that $\hat{\sigma}^{\rho}(\hat{A}, \nu)=0$. Therefore $\hat{\sigma}^{\rho}(\hat{A}, \nabla)=0$. Hence $\mathrm{e}^{\hat{\sigma}(a,-)}(\nabla)=\nabla$.

6.2. The case of free $\pi$. It is interesting to compute the image of the map $\rho \mapsto \nabla_{\rho}$ defined in Lemma 6.2. We do it here in the case where $\pi$ is a free group of finite rank $n$. Pick free generators $x_{1}, \ldots, x_{n}$ of $\pi$. We say that an element $a$ of $\hat{A}$ is non-degenerate if there is an invertible $n \times n$ matrix $\left(a_{r, s}\right)_{r, s}$ over $\mathbb{K}$ such that

$$
a-\operatorname{aûg}(a) \equiv \sum_{r, s=1}^{n}\left(x_{r}-1\right) a_{r, s}\left(x_{s}-1\right)\left(\bmod \hat{A}_{3}\right) .
$$

It is easy to check that the non-degeneracy of $a$ does not depend on the choice of the free generators $x_{1}, \ldots, x_{n}$ of $\pi$.

Lemma 6.5. Let $\pi$ be a finitely generated free group and $\nabla \in \hat{A}_{1}$. There is a nondegenerate F-pairing $\rho$ in $\hat{A}$ such that $\nabla_{\rho}=\nabla$ if and only if $\nabla$ is non-degenerate.

Proof. It is clear from (6.1.2) that $\nabla_{\rho} \in \hat{A}_{1}$ is non-degenerate for any non-degenerate F-pairing $\rho$ in $\hat{A}$. To prove the converse, pick free generators $x_{1}, \ldots, x_{n}$ of $\pi$. We first extend to $\hat{A}$ the Fox derivatives in $A$ mentioned in Remark 2.5 1. Using the interpretation of $\hat{A}$ in terms of formal power series mentioned in Section 3.1, one observes that any $a \in \hat{A}$ expands uniquely as aûg $(a)+\sum_{i} a_{i}\left(x_{i}-1\right)$ with $a_{i} \in \hat{A}$. We set $\partial_{i}(a)=a_{i}$. Similarly, any $a \in \hat{A}$ expands uniquely as aûg $(a)+\sum_{i}\left(x_{i}-1\right) a^{i}$ with $a^{i} \in \hat{A}$, and we set $\partial^{i}(a)=a^{i}$.

Suppose that $\nabla \in \hat{A}_{1}$ is non-degenerate. We can expand

$$
\nabla=\sum_{r, s=1}^{n}\left(x_{r}-1\right) c_{r, s}\left(x_{s}-1\right)
$$

where $c_{r, s} \in \hat{A}$ for all $r, s$. Though we shall not need it, note that $c_{r, s}=\partial^{r} \partial_{s}(\nabla)=$ $\partial_{s} \partial^{r}(\nabla)$. The non-degeneracy of $\nabla$ implies that the $n \times n$ matrix $\left(\operatorname{au} g\left(c_{r, s}\right)\right.$ ) is invertible over $\mathbb{K}$. Hence the matrix $\left(c_{r, s}\right)_{r, s}$ is invertible over $\hat{A}$. Let $\left(b_{r, s}\right)_{r, s}$ be the inverse matrix. Then the formula

$$
\rho(a, b)=\sum_{r, s=1}^{n} \partial_{r}(a) b_{r, s} \partial^{s}(b)
$$


defines a non-degenerate F-pairing $\rho$ in $\hat{A}$. By (6.1.2), we obtain that $\nabla_{\rho}=\nabla$.

Lemmas 6.2 and 6.5 show that any non-degenerate $\nabla \in \hat{A}_{1}$ determines a nondegenerate F-pairing $\rho=\rho_{\nabla}$ in $\hat{A}$. Then, any pair $(k \in \mathbb{K}$, a conjugacy class $\alpha$ in $\hat{\pi}$ such that $\alpha{ }_{\rho}{ }_{\rho} \alpha=0$ ) determines an automorphism $t_{k, \alpha}$ of $\hat{\pi}$. For example, we can pick a non-degenerate $\nu \in \hat{\pi} \subset \hat{A}$ and apply these constructions to $\nabla=\nu-1$. Theorem 6.4 ensures that all the twists $t_{k, \alpha}$ preserve $\nu$ and $\rho=\rho_{\nabla}$. Note as an additional bonus that the form ' $\rho$ in $H_{1}(\pi ; \mathbb{K})$ associated with $\nabla=\nu-1$ is skewsymmetric so that the twists $t_{k, \alpha}$ are defined for all pairs ( $k \in \mathbb{K}$, a conjugacy class $\alpha$ in $\hat{\pi}$ ). This gives a rich family of automorphisms of $\hat{\pi}$ preserving $\nu$. In particular, we obtain that all transvections in $H_{1}(\pi ; \mathbb{K})$ determined by ${ }^{\prime}$ lift to group automorphisms of $\hat{\pi}$ preserving $\nu$ and $\rho$.

6.3. Remark. The proof of Lemma 6.2 reproduces (in a more general setting) the arguments used in the proof of Lemma 2.11 in [Tu1].

\section{The Homotopy INTERSECTION FORM OF A SURFACE}

We discuss the homotopy intersection form of a surface [Tu1 and the associated derived form. In this section, $\Sigma$ is a smooth connected oriented surface with nonempty boundary and a base point $* \in \partial \Sigma$. We provide $\partial \Sigma$ with orientation induced by that of $\Sigma$. Set $\pi=\pi_{1}(\Sigma, *)$ and $A=\mathbb{K}[\pi]$.

7.1. Paths and loops. By paths and loops we shall mean piecewise-smooth paths and loops in $\Sigma$. The product $\alpha \beta$ of two paths $\alpha$ and $\beta$ is obtained by running first along $\alpha$ and then along $\beta$. Given two distinct simple (that is, multiplicity 1 ) points $p, q$ on a path $\alpha$, we denote by $\alpha_{p q}$ the path from $p$ to $q$ running along $\alpha$ in the positive direction. For a simple point $p$ of a loop $\alpha$ we denote by $\alpha_{p}$ the loop $\alpha$ based at $p$.

We shall use a second base point $\bullet \in \partial \Sigma \backslash\{*\}$ lying "slightly before" * on $\partial \Sigma$. We fix an embedded path $\nu_{\bullet *}$ running from $\bullet$ to $*$ along $\partial \Sigma$ in the positive direction, and we denote the inverse path by $\bar{\nu}_{* \bullet}$. The element of $\pi$ represented by a loop $\alpha$ based at $*$ is denoted $[\alpha]$. We say that a loop $\alpha$ based at $\bullet$ represents $\left[\bar{\nu}_{* \bullet} \alpha \nu_{\bullet *}\right] \in \pi$.

7.2. The homotopy intersection form $\eta$. The homotopy intersection form of $\Sigma$ is the $\mathbb{K}$-bilinear map $\eta: A \times A \rightarrow A$ defined, for any $a, b \in \pi$, by

$$
\eta(a, b)=\sum_{p \in \alpha \cap \beta} \varepsilon_{p}(\alpha, \beta)\left[\bar{\nu}_{* \bullet} \alpha_{\bullet p} \beta_{p *}\right] .
$$

Here, $\alpha$ is a loop based at $\bullet$ and representing $a$; $\beta$ is a loop based at $*$ and representing $b$. We assume that $\alpha$ and $\beta$ meet transversely in a finite set $\alpha \cap \beta$ of simple points of $\alpha, \beta$. Each crossing $p \in \alpha \cap \beta$ has a sign $\varepsilon_{p}(\alpha, \beta)= \pm 1$ which is +1 if and only if the frame (the positive tangent vector of $\alpha$ at $p$, the positive tangent vector of $\beta$ at $p$ ) is positively oriented.

It is easy to verify that $\eta$ is well-defined and is an F-pairing in the sense of Section 2.2. The associated T-pairing $\lambda$, defined by $\lambda(a, b)=\eta(a, b) b^{-1}$ for $a, b \in \pi$, first appeared in [Tu1] where it was used to characterize pairs of elements in $\pi$ that can be represented by non-intersecting loops, and to characterize (in the compact case) those automorphisms of $\pi$ that arise from self-diffeomorphisms of $\Sigma$. The pairing $\lambda$ also implicitly appeared in $\mathrm{Pa}$ in connection with Reidemeister's equivariant 
intersection forms on closed surfaces. Applications of $\lambda$ to mapping class groups are discussed in $[\mathrm{Pe}$.

Lemma 7.1. The homological form ${ }^{\eta}: H_{1}(\pi ; \mathbb{K}) \times H_{1}(\pi ; \mathbb{K}) \rightarrow \mathbb{K}$ induced by $\eta$ is the standard homological intersection pairing $\cdot$ in $H_{1}(\Sigma ; \mathbb{K})$.

Proof. Clearly, $H_{1}(\pi ; \mathbb{K}) \simeq H_{1}(\Sigma ; \mathbb{K})$. For any loops $\alpha, \beta$ as in the definition of $\eta$,

$$
[\alpha] \bullet_{\eta}[\beta]=\operatorname{aug}(\eta([\alpha],[\beta]))=\sum_{p \in \alpha \cap \beta} \varepsilon_{p}(\alpha, \beta)=[\alpha] \cdot[\beta] .
$$

Lemma 7.2. The (right) derived form $\sigma: A \times A \rightarrow A$ of $\eta$ is given, for any $a, b \in \pi, b y$

$$
\sigma(a, b)=\sum_{p \in \alpha \cap \beta} \varepsilon_{p}(\alpha, \beta)\left[\beta_{* p} \alpha_{p} \beta_{p *}\right]
$$

where $\alpha$ is a loop representing the conjugacy class of a and $\beta$ is a loop based at * and representing $b$ such that $\alpha, \beta$ meet transversely at a finite set of simple points.

Proof. Using standard local moves on loops generating the relation of homotopy, it is easy to check that the right-hand side of (7.2.2) is preserved under free homotopies of $\alpha$. We can therefore assume that $\alpha$ is based at $\bullet$ and represents $a$. Then

$$
\begin{aligned}
& \sigma(a, b)=b a^{\eta(a, b)} \\
& =\sum_{p \in \alpha \cap \beta} \varepsilon_{p}(\alpha, \beta) b\left[\bar{\nu}_{* \bullet} \alpha \bullet p \beta_{p *}\right]^{-1} a\left[\bar{\nu}_{* \bullet} \alpha_{\bullet} \beta_{p *}\right] \\
& =\sum_{p \in \alpha \cap \beta} \varepsilon_{p}(\alpha, \beta)\left[\beta\left(\beta^{-1}\right)_{* p}\left(\alpha^{-1}\right)_{p \bullet} \nu_{\bullet *} \bar{\nu}_{* \bullet} \alpha \nu_{\bullet *} \bar{\nu}_{* \bullet} \alpha_{\bullet} \beta_{p *}\right] \\
& =\sum_{p \in \alpha \cap \beta} \varepsilon_{p}(\alpha, \beta)\left[\beta_{* p}\left(\alpha^{-1}\right)_{p \bullet} \alpha \alpha_{\bullet p} \beta_{p *}\right] \\
& =\sum_{p \in \alpha \cap \beta} \varepsilon_{p}(\alpha, \beta)\left[\beta_{* p} \alpha_{p} \beta_{p *}\right] .
\end{aligned}
$$

The form (7.2.2) was first introduced by Kawazumi and Kuno [KK1]. They did not consider connections with the homotopy intersection form.

7.3. Properties of $\sigma$. The properties of the derived forms of F-pairings obtained in Section 2 fully apply to the form $\sigma=\sigma^{\eta}$. In particular, $\sigma(a,-)$ is a derivation of $A$ and $\sigma\left(c a c^{-1},-\right)=\sigma(a,-)$ for all $a \in A, c \in \pi$. We state two additional properties of $\sigma$.

Lemma 7.3. If the conjugacy classes of $a, b \in \pi$ can be represented by disjoint loops in $\Sigma$, then the derivations $\sigma\left(a^{m},-\right)$ and $\sigma\left(b^{n},-\right)$ commute for all $m, n \geq 0$.

Proof. If $a, b$ are represented by disjoint loops, then so are the powers of $a, b$. Therefore the claim of the lemma directly follows from Lemma 7.2

Lemma 7.4. For any $x \in I^{2} \subset A$ and $b, c \in \pi$,

$$
\eta(\sigma(x, b), c)+\eta(b, \sigma(x, c))=\sigma(x, \eta(b, c)) .
$$


Proof. Pick any $a \in \pi$. Let $\alpha$ be a loop representing the conjugacy class of $a$, let $\beta$ be a loop based at $\bullet$ and representing $b$, let $\gamma$ be a loop based at $*$ and representing $c$. We assume that these three loops meet transversely at simple points. Then

$$
\eta(\sigma(a, b), c)=\sum_{p \in \alpha \cap \beta} \varepsilon_{p}(\alpha, \beta) \eta\left(\left[\bar{\nu}_{* \bullet} \beta_{\bullet p} \alpha_{p} \beta_{p \bullet} \nu_{\bullet *}\right], c\right)=X+Y+Z
$$

where we have set

$$
\begin{aligned}
X & =\sum_{p \in \alpha \cap \beta} \sum_{q \in \alpha \cap \gamma} \varepsilon_{p}(\alpha, \beta) \varepsilon_{q}(\alpha, \gamma)\left[\bar{\nu}_{* \bullet} \beta_{\bullet} \alpha_{p q} \gamma_{q *}\right], \\
Y & =\sum_{p \in \alpha \cap \beta} \sum_{\substack{q \in \beta \cap \gamma \\
q<p \text { on } \beta}} \varepsilon_{p}(\alpha, \beta) \varepsilon_{q}(\beta, \gamma)\left[\bar{\nu}_{* \bullet} \beta_{\bullet} \gamma_{q *}\right], \\
Z & =\sum_{p \in \alpha \cap \beta} \sum_{\substack{q \in \beta \cap \gamma \\
q>p \text { on } \beta}} \varepsilon_{p}(\alpha, \beta) \varepsilon_{q}(\beta, \gamma)\left[\bar{\nu}_{* \bullet} \beta_{\bullet p} \alpha_{p} \beta_{p q} \gamma_{q *}\right] .
\end{aligned}
$$

Here we write $q<p$ (or $p>q$ ) on $\beta$ whenever $\beta$ passes first through the point $q$ and then through $p$. Similarly,

$$
\eta(b, \sigma(a, c))=\sum_{q \in \alpha \cap \gamma} \varepsilon_{q}(\alpha, \gamma) \eta\left(b,\left[\gamma_{* q} \alpha_{q} \gamma_{q *}\right]\right)=X^{\prime}+Y^{\prime}+Z^{\prime}
$$

where we have set

$$
\begin{aligned}
X^{\prime} & =\sum_{q \in \alpha \cap \gamma} \sum_{p \in \beta \cap \alpha} \varepsilon_{q}(\alpha, \gamma) \varepsilon_{p}(\beta, \alpha)\left[\bar{\nu}_{* \bullet} \beta_{\bullet} \alpha_{p q} \gamma_{q *}\right] \\
Y^{\prime} & =\sum_{q \in \alpha \cap \gamma} \sum_{\substack{p \in \beta \cap \gamma \\
p<q \text { on } \gamma}} \varepsilon_{q}(\alpha, \gamma) \varepsilon_{p}(\beta, \gamma)\left[\bar{\nu}_{* \bullet} \beta \beta_{\bullet} \gamma_{p q} \alpha_{q} \gamma_{q *}\right], \\
Z^{\prime} & =\sum_{q \in \alpha \cap \gamma} \sum_{\substack{p \in \beta \cap \gamma \\
p>q \text { on } \gamma}} \varepsilon_{q}(\alpha, \gamma) \varepsilon_{p}(\beta, \gamma)\left[\bar{\nu}_{* \bullet} \beta_{\bullet} \gamma_{p *}\right] .
\end{aligned}
$$

Moreover, we have

$$
\begin{aligned}
\sigma(a, \eta(b, c))= & \sum_{p \in \beta \cap \gamma} \varepsilon_{p}(\beta, \gamma) \sigma\left(a,\left[\bar{\nu}_{* \bullet} \beta_{\bullet p} \gamma_{p *}\right]\right) \\
= & \sum_{p \in \beta \cap \gamma} \sum_{\substack{q \in \alpha \cap \beta \\
q<p \text { on } \beta}} \varepsilon_{p}(\beta, \gamma) \varepsilon_{q}(\alpha, \beta)\left[\bar{\nu}_{* \bullet} \beta_{\bullet} \alpha_{q} \beta_{q p} \gamma_{p *}\right] \\
& +\sum_{p \in \beta \cap \gamma} \sum_{\substack{q \in \alpha \cap \gamma \\
q>p \text { on } \gamma}} \varepsilon_{p}(\beta, \gamma) \varepsilon_{q}(\alpha, \gamma)\left[\bar{\nu}_{* \bullet} \beta_{\bullet} \gamma_{p q} \alpha_{q} \gamma_{q *}\right]=Z+Y^{\prime} .
\end{aligned}
$$

Clearly, $X=-X^{\prime}$. Therefore

$$
\begin{aligned}
& \eta(\sigma(a, b), c)+\eta(b, \sigma(a, c))-\sigma(a, \eta(b, c))=Y+Z^{\prime} \\
= & \sum_{r \in \beta \cap \gamma}\left(\sum_{\substack{p \in \alpha \cap \beta \\
r<p \text { on } \beta}} \varepsilon_{p}(\alpha, \beta)+\sum_{\substack{q \in \alpha \cap \gamma \\
r>q \text { on } \gamma}} \varepsilon_{q}(\alpha, \gamma)\right) \varepsilon_{r}(\beta, \gamma)\left[\bar{\nu}_{* \bullet} \beta_{\bullet r} \gamma_{r *}\right] \\
= & \sum_{r \in \beta \cap \gamma}\left([\alpha] \cdot\left[\beta_{r \bullet} \nu_{\bullet *} \gamma_{* r}\right]\right) \varepsilon_{r}(\beta, \gamma)\left[\bar{\nu}_{* \bullet} \beta_{\bullet r} \gamma_{r *}\right] .
\end{aligned}
$$


Denoting the right-hand side by $R(a)$ we observe that the resulting map $R: \pi \rightarrow A$ satisfies $R\left(a a^{\prime}\right)=R(a)+R\left(a^{\prime}\right)$ for all $a, a^{\prime} \in \pi$. We deduce that (7.3.1) holds true for $x=(a-1)\left(a^{\prime}-1\right)$. The conclusion follows.

7.4. Remarks. 1. It is easy to show that the F-pairing $\eta$ is weakly skew-symmetric in the sense of Section 2.4. More precisely, for any $a, b \in A$,

$$
\eta(a, b)+\bar{\eta}(a, b)=-(a-\operatorname{aug}(a))(b-\operatorname{aug}(b)) .
$$

(An equivalent formula in terms of the associated T-pairing is stated in Tu1, Formula (5).) This implies that the left derived form $A \times A \rightarrow A$ of $\eta$ is given by $(a, b) \mapsto-\sigma(b, a)$ for any $a, b \in A$.

2. By Remark 2.5 2, the right derived form $\sigma$ of $\eta$ induces a $\mathbb{K}$-bilinear form $q \sigma: \mathbb{K}[\tilde{\pi}] \times \mathbb{K}[\check{\pi}] \rightarrow \mathbb{K}[\check{\pi}]$. Lemma 7.2 implies that for any $a, b \in \check{\pi}$ represented by transversal free loops $\alpha, \beta$, we have

$$
q \sigma(a, b)=\sum_{p \in \alpha \cap \beta} \varepsilon_{p}(\alpha, \beta)\left[\alpha_{p} \beta_{p}\right] .
$$

Thus, $q \sigma$ is the Lie bracket in $\mathbb{K}[\check{\pi}]$ introduced by Goldman Go. Note that the map $\mathbb{K}[\check{\pi}] \rightarrow \operatorname{Der}(A)$ defined by $a \mapsto \sigma(a,-)$ is a Lie algebra homomorphism from Goldman's Lie algebra to the Lie algebra of derivations in $A$, see [KK1].

\section{The EXTENDED MAPPING CLASS GRoup AND THE TWISTS}

We apply the constructions and results of Sections 4 and 5 to the homotopy intersection form of a surface. As above, $\Sigma$ is a smooth connected oriented surface with non-empty boundary, $* \in \partial \Sigma, \pi=\pi_{1}(\Sigma, *)$, and $A=\mathbb{K}[\pi]$.

8.1. The extended mapping class group of $\Sigma$. Recall the complete Hopf algebra $\hat{A}=\lim A / I^{m}$ where $I=\operatorname{Ker}$ aug $\subset A$, and the group $\operatorname{Aut}(\hat{A})$ consisting of H-automorphisms of $\hat{A}$ (see Section 5.1). Since $\pi$ is a free group, the natural homomorphism $\iota: A \rightarrow \hat{A}$ is injective, and we can view $A$ as a subalgebra of $\hat{A}$.

By Section 3.3, the homotopy intersection form $\eta$ in $A$ induces an F-pairing $\hat{\eta}: \hat{A} \times \hat{A} \rightarrow \hat{A}$. Let $\widehat{\mathcal{M}}(\Sigma, *) \subset \operatorname{Aut}(\hat{A})$ be the group of H-automorphisms of $\hat{A}$ that preserve $\hat{\eta}$. We call $\widehat{\mathcal{M}}(\Sigma, *)$ the extended mapping class group of $(\Sigma, *)$. Lemma 3.4 implies that all elements of $\widehat{\mathcal{M}}(\Sigma, *)$ preserve the derived form $\hat{\sigma}$ of $\hat{\eta}$.

We now relate $\widehat{\mathcal{M}}(\Sigma, *)$ to the classical mapping class group $\mathcal{M}(\Sigma, *)$ of $(\Sigma, *)$, defined as the group of isotopy classes of orientation-preserving self-diffeomorphisms of $\Sigma$ that fix $*$. We emphasize that, in this definition, the self-diffeomorphisms of $\Sigma$ are not required to be the identity on $\partial \Sigma$. In particular, the Dehn twist about a closed curve parallel to a circle component of $\partial \Sigma \backslash\{*\}$ is trivial in $\mathcal{M}(\Sigma, *)$.

Any orientation-preserving diffeomorphism $f:(\Sigma, *) \rightarrow(\Sigma, *)$ induces an automorphism of $\pi$, which extends by $\mathbb{K}$-linearity to an automorphism $\zeta(f)$ of $A=\mathbb{K}[\pi]$. The latter preserves the $I$-filtration and extends uniquely to an H-automorphism $\hat{\zeta}(f)$ of $\hat{A}$. The automorphism $\hat{\zeta}(f)$ preserves $\hat{\eta}$ because $\zeta(f)$ preserves $\eta$. Thus the formula $f \mapsto \hat{\zeta}(f)$ defines a group homomorphism $\hat{\zeta}: \mathcal{M}(\Sigma, *) \rightarrow \widehat{\mathcal{M}}(\Sigma, *)$.

Theorem 8.1. If all components of $\partial \Sigma$ are circles, then $\hat{\zeta}$ is an injection.

Proof. Since $\pi \subset A \subset \hat{A}$, any $f \in \operatorname{Ker} \hat{\zeta}$ induces the identity automorphism of $\pi$. The surface $\Sigma$ is a $K(\pi, 1)$-space since the homology of its universal covering is zero in all positive degrees. Therefore $f$ is homotopic to $\mathrm{id}_{\Sigma}$ relatively to $*$. It 
is known that homotopic diffeomorphisms of $(\Sigma, *)$ are isotopic, see, for instance, $\mathrm{Ep}$, Theorem 6.4. We conclude that $f=1 \in \mathcal{M}(\Sigma, *)$.

Theorem 8.1]justifies the term "extended mapping class group" for $\widehat{\mathcal{M}}(\Sigma, *)$. The assumption on $\partial \Sigma$ probably can be removed, the missing part is the equivalence of homotopy and isotopy without this assumption. In the case where $\Sigma$ is compact and $\partial \Sigma \cong S^{1}$, the group $\widehat{\mathcal{M}}(\Sigma, *)$ is closely related to the extensions of $\mathcal{M}(\Sigma, *)$ introduced in the study of homology cobordisms of $\Sigma$ in terms of automorphisms of the pronilpotent completion (or the Malcev completion) of $\pi$, see GL, $\mathrm{Ha}, \mathrm{Mo}$.

8.2. Generalized Dehn twists. Each closed curve $C$ in $\Sigma$ determines an element $c$ of $\pi$ defined up to inversion and conjugation. For $k \in \mathbb{K}$, the generalized Dehn twist along $C$ with parameter $k$ is

$$
t_{k, C}=\mathrm{e}^{\hat{\sigma}\left(k \log ^{2}(c),-\right)}: \hat{A} \rightarrow \hat{A} .
$$

When $\Sigma$ is compact, $\partial \Sigma \cong S^{1}$, and $k=1 / 2$, (8.2.1) is equivalent to the definition of a generalized Dehn twist given by Kuno [Ku] ;e explain this in Section 10 .

The results of Section 4.3 and Theorem 5.1 imply that $t_{k, C}$ is an H-automorphism of $\hat{A}$ independent of the choice of $c$. By definition, $t_{k, C}$ is preserved under homotopies of the curve $C$ in $\Sigma$.

Lemma 8.2. $t_{k, C} \in \widehat{\mathcal{M}}(\Sigma, *)$ for all $k, C$.

Proof. We need only to prove that $t_{k, C}$ preserves $\hat{\eta}$. Consider an arbitrary weakly nilpotent $\mathbb{K}$-linear homomorphism $d: \hat{A} \rightarrow \hat{A}$ such that for all $a, b \in \hat{A}$,

$$
d(\hat{\eta}(a, b))=\hat{\eta}(d(a), b)+\hat{\eta}(a, d(b)) .
$$

Then $\mathrm{e}^{d}$ preserves $\hat{\eta}$ : indeed we have for all $a, b \in \hat{A}$,

$$
\begin{aligned}
\mathrm{e}^{d}(\hat{\eta}(a, b)) & =\sum_{r \geq 0} \frac{d^{r}}{r !}(\hat{\eta}(a, b)) \\
& =\sum_{r \geq 0} \frac{1}{r !} \sum_{i=0}^{r}\left(\begin{array}{l}
r \\
i
\end{array}\right) \hat{\eta}\left(d^{i}(a), d^{r-i}(b)\right) \\
& =\sum_{i \geq 0} \sum_{j \geq 0} \frac{1}{i ! j !} \hat{\eta}\left(d^{i}(a), d^{j}(b)\right)=\hat{\eta}\left(\mathrm{e}^{d}(a), \mathrm{e}^{d}(b)\right) .
\end{aligned}
$$

Lemma 7.4 shows that $d=\hat{\sigma}\left(k \log ^{2}(c),-\right): \hat{A} \rightarrow \hat{A}$ satisfies the identity (8.2.2). Hence, $t_{k, C}=\mathrm{e}^{d}$ preserves $\hat{\eta}$.

We now show that for a simple closed curve $C \subset \Sigma$, the automorphism $t_{1 / 2, C}$ of $\hat{A}$ is induced by the classical Dehn twist $T_{C}: \Sigma \rightarrow \Sigma$ about $C$. Recall that $T_{C}$ is a diffeomorphism supported in a regular neighborhood of $C$ and acting on an arc meeting $C$ transversely in one point as shown in Figure 1 .

The following theorem generalizes a theorem of Kawazumi and Kuno [KK1] concerning the case of a compact surface bounded by a circle. In particular, Kawazumi and Kuno were the first to understand the role of the formal series $\log ^{2}(x) / 2$ in the computation of the action of $T_{C}$ on the fundamental group of such a surface.

Theorem 8.3. If $C \subset \Sigma$ is a simple closed curve, then

$$
t_{1 / 2, C}=\hat{\zeta}\left(T_{C}\right) .
$$



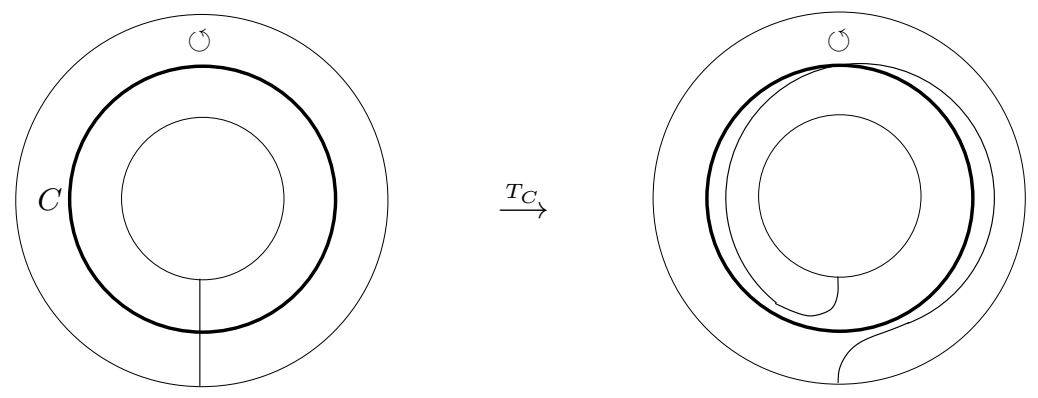

Figure 1. The action of the Dehn twist $T_{C}$ in a regular neighborhood of a simple closed curve $C$.

Proof. Our proof is inspired by the Kawazumi-Kuno [KK1] arguments though we avoid their use of symplectic expansions. We first reduce the theorem to the case of compact surfaces. Pick any $\beta \in \pi$ and represent $\beta$ by an immersed loop in $\Sigma$ based at $*$. Pick a compact connected surface $\Sigma^{\prime} \subset \Sigma$ whose interior contains this loop, $C$, and an $\operatorname{arc} \gamma$ connecting $*$ to a point of $C$. (We do not assume that $\partial \Sigma^{\prime} \subset \partial \Sigma$.) Set $\pi^{\prime}=\pi_{1}\left(\Sigma^{\prime}, *\right), A^{\prime}=\mathbb{K}\left[\pi^{\prime}\right]$, and let $\hat{A}^{\prime}$ be the fundamental completion of $A^{\prime}$. The inclusion $\Sigma^{\prime} \subset \Sigma$ induces an algebra homomorphism $i: \hat{A}^{\prime} \rightarrow \hat{A}$. Let $c^{\prime} \in \pi^{\prime}$ and $c \in \pi$ be represented by the loop $\gamma C \gamma^{-1}$ where $C$ is oriented in an arbitrary way. It is clear that the following two diagrams commute:
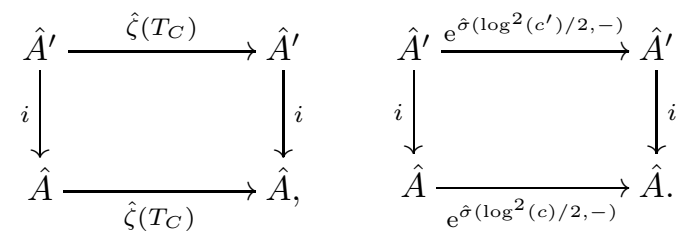

So, if $\hat{\zeta}\left(T_{C}\right)=t_{1 / 2, C}$ in $\operatorname{Aut}\left(\hat{A}^{\prime}\right)$, then $\hat{\zeta}\left(T_{C}\right)=t_{1 / 2, C}$ on $i\left(\hat{A}^{\prime}\right) \subset \hat{A}$. Clearly, $\beta \in i\left(\hat{A}^{\prime}\right)$. Thus, if the theorem holds for all compact surfaces, then $\hat{\zeta}\left(T_{C}\right)=t_{1 / 2, C}$ on $\pi \subset \hat{A}$. It remains to observe that any two filtered automorphisms of $\hat{A}$ coinciding on $\pi$ are equal. Indeed, they induce the same automorphism of $\hat{A} / \hat{A}_{m} \simeq A / I^{m}$ for all integer $m \geq 1$.

Assume that $\Sigma$ is compact. Denote the component of $\partial \Sigma$ containing the base point $*$ by $\partial_{*}$, and connect $*$ to $C$ by an embedded $\operatorname{arc} \gamma \subset \Sigma$ meeting $C$ solely at the terminal endpoint. A regular neighborhood $P \subset \Sigma$ of $\partial_{*} \cup \gamma \cup C$ is a pair of pants, see Figure 2. The components of $\partial P$ distinct from $\partial_{*}$ are $\alpha$ (which is parallel to $C$ ) and $\alpha^{\prime}$. Two situations may occur: either $\Sigma \backslash P$ is connected $(C$ is non-separating) or $\Sigma \backslash P$ has two connected components ( $C$ is separating). In both cases we orient $C$ as shown in Figure 2 and let $c=\left[\gamma C \gamma^{-1}\right] \in \pi$.

We first consider the case of separating $C$. Let $L$ and $D$ be the components of $\Sigma \backslash \operatorname{int}(P)$ that contain $\alpha$ and $\alpha^{\prime}$ respectively. Applying the van Kampen theorem, we can find a basis $\Lambda \cup \Delta$ of $\pi$ such that each $\lambda \in \Lambda$ (respectively $\delta \in \Delta$ ) is represented by a loop in $L$ (respectively, in $D$ ) transported to the base point * along an arc in $\Sigma$ which meets $\alpha$ (respectively $\alpha^{\prime}$ ) transversely in one point, see Figure 2. The action of $T_{C}$ on $\Lambda \cup \Delta$ is computed by

$$
T_{C}(\lambda)=c \lambda c^{-1} \quad \text { for all } \lambda \in \Lambda \quad \text { and } \quad T_{C}(\delta)=\delta \quad \text { for all } \delta \in \Delta .
$$



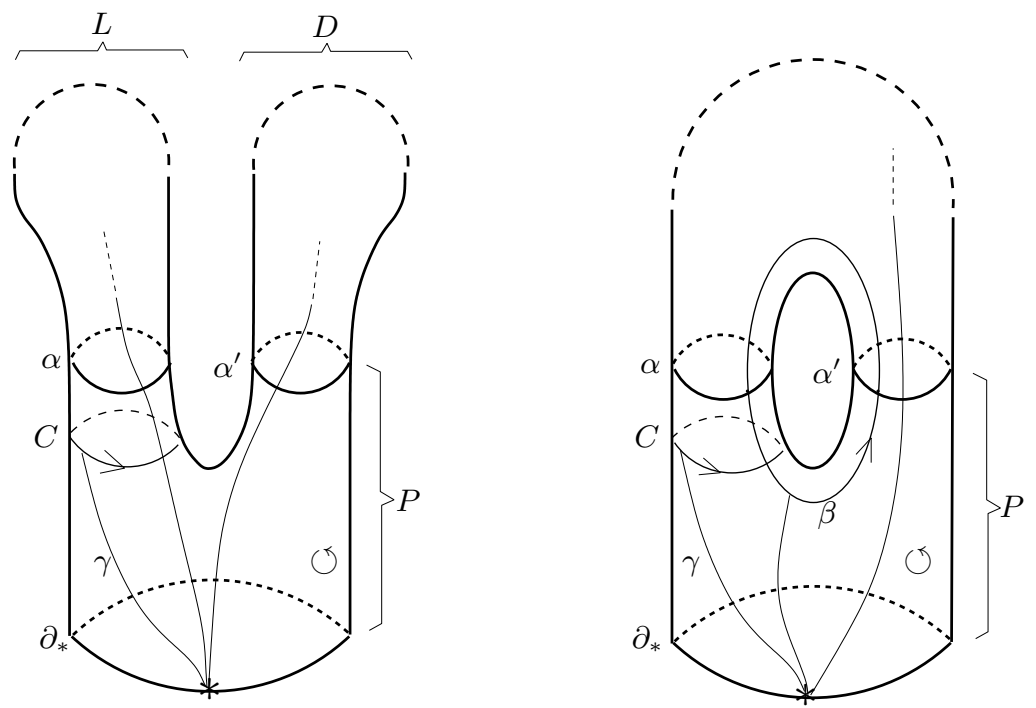

FiguRE 2. The separating case and the non-separating case.

We now compute the derivation $d=\hat{\sigma}\left(\log ^{2}(c) / 2,-\right)$ of the algebra $\hat{A}$. Clearly, $\eta(c, \lambda)=\lambda-1$ for all $\lambda \in \Lambda$. Formula (5.2.1) with $k=1 / 2$ gives

$$
d(\lambda)=\lambda\left(\lambda^{-1} \log (c) \lambda-\log (c)\right)=\log (c) \lambda-\lambda \log (c)=[\log (c), \lambda] .
$$

Here the commutator $a b-b a$ of two elements of an algebra is denoted by $[a, b]$. Thus, the derivations $d$ and $[\log (c),-]$ of $\hat{A}$ are equal on $\Lambda$. Since $c$ belongs to the subgroup of $\pi$ generated by $\Lambda$, we deduce that

$$
d(\log (c))=[\log (c), \log (c)]=0 .
$$

This formula implies by induction on $n \geq 1$ that for all $\lambda \in \Lambda$,

$$
d^{n}(\lambda)=[\log (c),-]^{n}(\lambda) .
$$

Therefore

$$
t_{1 / 2, C}(\lambda)=\mathrm{e}^{d}(\lambda)=\mathrm{e}^{[\log (c),-]}(\lambda)=\mathrm{e}^{\log (c)} \lambda \mathrm{e}^{-\log (c)}=c \lambda c^{-1}
$$

(cf. Appendix A). Since $C$ does not meet the loops representing the elements of $\Delta$, the automorphism $t_{1 / 2, C}$ fixes $\Delta$ pointwise. Comparing with (8.2.4) we obtain that $\hat{\zeta}\left(T_{C}\right)=t_{1 / 2, C}$ on $\Lambda \cup \Delta$. Since $\Lambda \cup \Delta$ generates $\pi$, we have $\hat{\zeta}\left(T_{C}\right)=t_{1 / 2, C}$.

If $C$ is non-separating, then there is a simple closed curve in $\Sigma$ meeting both $\alpha$ and $\alpha^{\prime}$ transversely in one point. We orient this curve and transport it to $*$ along an embedded arc in $P \backslash(C \cup \gamma)$ as in Figure 2, Let $\beta$ be the element of $\pi=\pi_{1}(\Sigma, *)$ represented by the resulting loop. By the van Kampen theorem, the group $\pi$ is generated by $\{c, \beta\} \cup \Theta$ where $\Theta \subset \pi$ is a set whose elements are represented by loops in $\Sigma \backslash P$ transported to $*$ along an arc in $\Sigma \backslash C$ meeting $\alpha^{\prime}$ transversely in one point, see Figure 2. The action of $T_{C}$ on $\{c, \beta\} \cup \Theta$ is computed by

$$
T_{C}(\theta)=\theta \text { for all } \theta \in\{c\} \cup \Theta \text { and } T_{C}(\beta)=\beta c^{-1} .
$$

We now compute the derivation $d=\hat{\sigma}\left(\log ^{2}(c) / 2,-\right)$ of $\hat{A}$ and the automorphism $t_{1 / 2, C}=\mathrm{e}^{d}$ of $\hat{A}$. The curve $C$ does not meet the loops representing the elements of $\Theta$. Therefore $d(\Theta)=0$ and $t_{1 / 2, C}$ fixes $\Theta$ pointwise. The curve $C$ may be pushed 
into $\Sigma \backslash P$ and the resulting curve is disjoint from $\gamma C \gamma^{-1}$. Therefore $d(c)=0$ and $t_{1 / 2, C}(c)=c$. Since $\eta(c, \beta)=-1$, Formula (5.2.1) with $k=1 / 2$ gives $d(\beta)=$ $-\beta \log (c)$. The equality $d(\log (c))=0$ implies that $d^{n}(\beta)=\beta(-\log (c))^{n}$ for all $n \geq 0$. Hence

$$
t_{1 / 2, C}(\beta)=\mathrm{e}^{d}(\beta)=\beta \mathrm{e}^{-\log (c)}=\beta c^{-1} .
$$

Comparing with (8.2.5), we conclude that $\hat{\zeta}\left(T_{C}\right)=t_{1 / 2, C}$ on the generating set $\{c, \beta\} \cup \Theta$ of $\pi$. Hence $\hat{\zeta}\left(T_{C}\right)=t_{1 / 2, C}$ on $\hat{A}$.

8.3. Properties of $t_{k, C}$. The properties of the twists stated in Section 4.3 specialize to the topological setting and imply the following properties of $t_{k, C}$ for any closed curve $C$ in $\Sigma$. First of all, the family $\left(t_{k, C}\right)_{k \in \mathbb{K}}$ is a one-parameter subgroup of $\widehat{\mathcal{M}}(\Sigma, *)$. This family is natural with respect to self-diffeomorphisms of $\Sigma$ : for any $f \in \mathcal{M}(\Sigma, *)$, we have $t_{k, f(C)}=\hat{\zeta}(f) t_{k, C} \hat{\zeta}(f)^{-1}$. To proceed, fix $k \in \mathbb{K}$.

(1) For all $m \geq 0$, we have $t_{k, C^{m}}=t_{k m^{2}, C}=\left(t_{k, C}\right)^{m^{2}}$ where $C^{m}$ is a closed curve winding $m$ times around $C$.

(2) For all $m \geq 1$, the automorphism of $\hat{A} / \hat{A}_{m} \simeq A / I^{m}$ induced by $t_{k, C}$ depends only on the conjugacy class in $\pi / \pi_{m}$ represented by $C$.

(3) If $\beta \in \pi$ can be represented by a loop disjoint from $C$, then $t_{k, C}(\beta)=\beta$. If $C^{\prime}$ is a loop in $\Sigma$ disjoint from $C$ then $t_{k, C}$ commutes with $t_{k^{\prime}, C^{\prime}}$ for all $k^{\prime} \in \mathbb{K}$. This follows from Lemma 7.3 .

(4) The automorphism of $H=\hat{A}_{1} / \hat{A}_{2} \simeq H_{1}(\Sigma ; \mathbb{K})$ induced by $t_{k, C}$ is the transvection $h \mapsto h+2 k([C] \cdot h)[C], h \in H$.

(5) Consider the quotient group $\pi /\langle c\rangle$ where $c$ is an element of $\pi$ represented by $C$ and $\langle c\rangle$ is the normal subgroup of $\pi$ generated by $c$. Let $K \subset \hat{A}$ be the kernel of the algebra homomorphism $\hat{A} \rightarrow \widehat{\mathbb{K}[\pi /\langle c\rangle]}$ induced by the projection $\pi \rightarrow \pi /\langle c\rangle$. Then $t_{k, C}(\beta) \in \beta K$ for all $\beta \in \pi$. This follows from the inclusions $\hat{\sigma}\left(c^{n}, \beta\right) \in \beta K$ for all $n \geq 1$.

Note the following corollary concerning the classical Dehn twist about a simple closed curve $C \subset \Sigma$ : for any $m \geq 1$, the action of $T_{C}$ on $\pi / \pi_{m}$ depends only on the conjugacy class in $\pi / \pi_{m}$ represented by $C$. This follows from Theorem 8.3 , Property (2) above, and the injectivity of the map $\pi / \pi_{m} \rightarrow A / I^{m}$ induced by the inclusion $\pi \subset A$. This property of $T_{C}$ was first established by Kawazumi and Kuno KK1 for compact $\Sigma$ with $\partial \Sigma \cong S^{1}$.

Finally, we formulate the naturality of the twists with respect to embeddings of surfaces. Suppose that $\Sigma$ is a subsurface of a surface $\Sigma^{+}$and the orientation of $\Sigma$ extends to $\Sigma^{+}$. Let $\gamma$ be a path in $\Sigma^{+} \backslash \bar{\Sigma}$ leading from $* \in \partial \Sigma$ to a point $*^{+} \in \partial \Sigma^{+}$. Transporting loops in $\Sigma$ along $\gamma$ we obtain a group homomorphism $\pi=\pi_{1}(\Sigma, *) \rightarrow \pi_{1}\left(\Sigma^{+}, *^{+}\right)=\pi^{+}$. Let $\gamma_{\#}: \hat{A} \rightarrow \hat{A}^{+}$be the induced algebra homomorphism where $\hat{A}^{+}$is the fundamental completion of $A^{+}=\mathbb{K}\left[\pi^{+}\right]$. Then for any $k \in \mathbb{K}$ and any closed curve $C$ in $\Sigma$, the following diagram commutes:

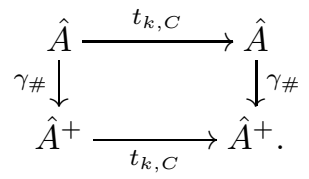

Here to define $t_{k, C}: \hat{A}^{+} \rightarrow \hat{A}^{+}$we view $C$ as a closed curve in $\Sigma^{+}$via the inclusion $\Sigma \subset \Sigma^{+}$. The commutativity of this diagram follows from the definitions and the 
equality $\gamma_{\#} \hat{\sigma}=\hat{\sigma}^{+}\left(\gamma_{\#} \times \gamma_{\#}\right): \hat{A} \times \hat{A} \rightarrow \hat{A}^{+}$where $\hat{\sigma}$ and $\hat{\sigma}^{+}$are the completed derived forms of the homotopy intersection forms in $\Sigma$ and $\Sigma^{+}$, respectively. The equality in question follows from Lemma 7.2

8.4. Example. Let $\Sigma$ be a 2-punctured disk with base point $* \in \partial \Sigma$ and let $C$ be the "figure eight" closed curve in $\Sigma$ as in Figure 3. We show that for any nonzero $k \in \mathbb{K}$, the twist $t_{k, C} \in \widehat{\mathcal{M}}(\Sigma, *)$ does not lie in the classical mapping class group $\mathcal{M}(\Sigma, *)$ or, more precisely, does not lie in the image of the homomorphism $\hat{\zeta}: \mathcal{M}(\Sigma, *) \rightarrow \widehat{\mathcal{M}}(\Sigma, *)$. A similar result was obtained by Kuno $\mathrm{Ku}$ for a "figure eight" curve in a compact surface with boundary $S^{1}$.

The group $\pi=\pi_{1}(\Sigma, *)$ is free on the generators $\alpha$ and $\beta$ shown in Figure 3 . The group $\mathcal{M}(\Sigma, *)$ is an infinite cyclic group generated by the half-twist $\tau$ about the dashed arc in Figure 3. This half-twist exchanges $\alpha$ and $\beta$ on the homological level. On the other hand, the isomorphism induced by $t_{k, C}$ in $\hat{A}_{1} / \hat{A}_{2} \simeq H_{1}(\Sigma ; \mathbb{K})$ is the identity since the homological intersection form of $\Sigma$ is trivial (here we use assertion (4) of Section 8.3). Consequently, $t_{k, C}$ is not an odd power of $\hat{\zeta}(\tau)$. To show that $t_{k, C} \neq \hat{\zeta}\left(\tau^{2 \ell}\right)$ for $\ell \in \mathbb{Z}$, observe that both endomorphisms $t_{k, C}$ and $\hat{\zeta}\left(\tau^{2 \ell}\right)$ of $\hat{A}$ act as the identity on $\hat{A} / \hat{A}_{2}$ and therefore have well-defined logarithms in the class of endomorphisms of $\hat{A}$. It is enough to prove that $\log \left(t_{k, C}\right) \neq \log \left(\hat{\zeta}\left(\tau^{2 \ell}\right)\right)$.

The loop $C$, oriented as in Figure 3 and transported to $*$ along the arc $\gamma$ represents $c=\alpha \beta^{-1} \in \pi$. We have $\eta(c, \alpha)=c-1+\alpha^{2}-c \alpha$ and (5.2.1) gives

$$
\log \left(t_{k, C}\right)(\alpha)=\hat{\sigma}\left(k \log ^{2}(c), \alpha\right)=2 k\left(\alpha^{-1} \log (c) \alpha-\log (c)\right) \alpha=2 k\left[\log \left(\alpha^{-1} c \alpha\right), \alpha\right]
$$

Since $\alpha^{-1} c \alpha=\beta^{-1} \alpha$, we have $\log \left(t_{k, C}\right)(\alpha)=2 k\left[\log \left(\beta^{-1} \alpha\right), \alpha\right]$. To compute the latter expression, we identify the completion $\hat{A}$ of $A=\mathbb{K}[\pi]$ with the algebra $\mathbb{K}\langle\langle a, b\rangle\rangle$ of formal power series in two non-commuting variables $a, b$ so that $\alpha=\mathrm{e}^{a}$ and $\beta=\mathrm{e}^{b}$. By the Baker-Campbell-Hausdorff formula,

$$
\begin{aligned}
\log \left(t_{k, C}\right)(\alpha) & =2 k\left[\log \left(\mathrm{e}^{-b} \mathrm{e}^{a}\right), \mathrm{e}^{a}\right]=2 k\left[\log \left(\mathrm{e}^{-b} \mathrm{e}^{a}\right), \mathrm{e}^{a}-1\right] \\
& =-2 k\left[b+\frac{1}{2}[b, a]-\frac{1}{12}[b,[b, a]]+\frac{1}{12}[a,[a, b]], a+\frac{a^{2}}{2}+\frac{a^{3}}{6}\right]+\cdots
\end{aligned}
$$

where the dots stand for the terms of total degree $\geq 5$ in $a, b$.

Clearly, $\tau^{2}$ is the Dehn twist about a simple closed curve in $\operatorname{Int}(\Sigma)$ parallel to $\partial \Sigma$. Hence $\hat{\zeta}\left(\tau^{2}\right)(x)=\nu x \nu^{-1}$ for all $x \in \pi$, where $\nu=\beta \alpha$. Therefore $\hat{\zeta}\left(\tau^{2}\right)=\mathrm{e}^{[\log (\nu),-]}$, cf. Appendix A. (Note that $\log (\nu) \in \hat{A}_{1}$ so that the derivation $x \mapsto[\log (\nu), x]$ of $\hat{A}$ is weakly nilpotent and has a well-defined exponential.) We have $\hat{\zeta}\left(\tau^{2 \ell}\right)=\left(\hat{\zeta}\left(\tau^{2}\right)\right)^{\ell}=$ $\mathrm{e}^{\ell[\log (\nu),-]}$ and

$$
\begin{aligned}
\log \left(\hat{\zeta}\left(\tau^{2 \ell}\right)\right)(\alpha) & =\ell\left[\log \left(\mathrm{e}^{b} \mathrm{e}^{a}\right), \mathrm{e}^{a}\right]=\ell\left[\log \left(\mathrm{e}^{b} \mathrm{e}^{a}\right), \mathrm{e}^{a}-1\right] \\
& =\ell\left[b+\frac{1}{2}[b, a]+\frac{1}{12}[b,[b, a]]+\frac{1}{12}[a,[a, b]], a+\frac{a^{2}}{2}+\frac{a^{3}}{6}\right]+\cdots
\end{aligned}
$$

Comparing with the expression for $\log \left(t_{k, C}\right)(\alpha)$, we deduce that $t_{k, C} \neq \hat{\zeta}\left(\tau^{2 \ell}\right)$.

8.5. Remarks. 1. We have $\widehat{\mathcal{M}}(\Sigma, *) \simeq \lim _{\longleftarrow} \operatorname{Aut}_{\eta}\left(A / I^{m}\right)$ where for every $m \geq 1$, $\operatorname{Aut}_{\eta}\left(A / I^{m}\right)$ is the group of Hopf algebra automorphisms $\varphi$ of $A / I^{m}$ such that 


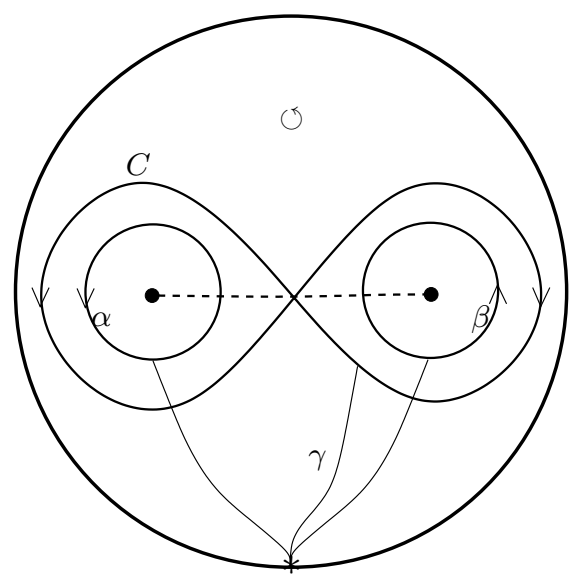

Figure 3. The "figure eight" curve $C$ on a 2-punctured disk.

$\varphi\left(I^{k} / I^{m}\right)=I^{k} / I^{m}$ for $k=1,2, \ldots, m$ and the following diagram commutes:

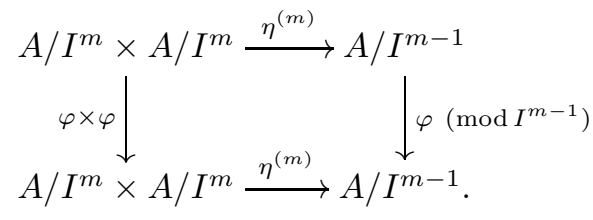

Here $\eta^{(m)}$ is the pairing induced by $\eta$, cf. Section 3.3. When $\mathbb{K}=\mathbb{R}$ and $\Sigma$ is compact, the group $\operatorname{Aut}_{\eta}\left(A / I^{m}\right)$ is a finite-dimensional Lie group and $\widehat{\mathcal{M}}(\Sigma, *)$ is a projective limit of such groups.

2. Fox pairings naturally arise in knot theory. Given a knot $K \subset S^{3}$, there is a canonical weakly skew-symmetric F-pairing in the fundamental completion of $\mathbb{Q}[\pi]$, where $\pi$ is the commutator subgroup of the knot group $\pi_{1}\left(S^{3} \backslash K\right)$, see Tu1, Appendix 3 or Tu2, Theorem E. We can apply the theory of twists introduced above in this setting. In this way, every conjugacy class in $\pi$ or, more generally, in $\hat{\pi}$ determines a 1-parameter family of group automorphisms of $\hat{\pi}$.

3. The definitions of the homotopy intersection form on surfaces and of the generalized Dehn twists have their analogues for non-orientable surfaces. We plan to discuss the non-orientable case elsewhere.

\section{TWISTS ON ARBITRARY ORIENTED SURFACES}

In this section we extend the definition of the generalized Dehn twists to closed curves on an arbitrary connected oriented surface $\Sigma$ (possibly with $\partial \Sigma=\emptyset$ ).

9.1. The group $\operatorname{Out}(\hat{A})$. The classical mapping class group $\mathcal{M}(\Sigma)$ of $\Sigma$ is defined as the group of isotopy classes of orientation-preserving diffeomorphisms $\Sigma \rightarrow \Sigma$. This group is related to the group of outer automorphisms of the fundamental group of $\Sigma$. Namely, pick any point $*$ in $\Sigma$ and set $\pi=\pi_{1}(\Sigma, *)$. Any self-diffeomorphism $f$ of $\Sigma$ induces an automorphism $f_{\#}$ of $\pi$ carrying the homotopy class $[\alpha]$ of a loop $\alpha$ based at $*$ to $\left[\gamma(f \circ \alpha) \gamma^{-1}\right]$, where $\gamma$ is a fixed path from $*$ to $f(*)$. The indeterminacy in the choice of $\gamma$ results in the fact that $f_{\#}$ is well-defined only up to conjugation by elements of $\pi$. In other words, $f_{\#} \in \operatorname{Out}(\pi)=\operatorname{Aut}(\pi) / \operatorname{Inn}(\pi)$. The 
formula $f \mapsto f_{\#}$ defines a homomorphism $\zeta: \mathcal{M}(\Sigma) \rightarrow$ Out $(\pi)$. If all components of $\partial \Sigma$ are circles, then $\zeta$ is injective, cf. Theorem 8.1. If $\Sigma$ is a closed surface then the image of $\zeta$ consists of all elements of $\operatorname{Out}(\pi)$ acting as the identity in $H_{2}(\pi ; \mathbb{Z}) \simeq \mathbb{Z}$.

Consider now the group algebra $A=\mathbb{K}[\pi]$ and its fundamental completion $\hat{A}=$ $\lim _{\longleftarrow} A / I^{m}$. An automorphism of $\hat{A}$ is inner if it is the conjugation by an element of $\hat{\pi} \subset \hat{A}$. The inner automorphisms of $\hat{A}$ form a normal subgroup $\operatorname{Inn}(\hat{A})$ of the $\operatorname{group} \operatorname{Aut}(\hat{A})$ of $\mathrm{H}$-automorphisms of $\hat{A}$. The outer automorphism group of $\hat{A}$ is the quotient group

$$
\operatorname{Out}(\hat{A})=\operatorname{Aut}(\hat{A}) / \operatorname{Inn}(\hat{A})
$$

Since the restriction map $\operatorname{Aut}(\hat{A}) \rightarrow \operatorname{Aut}(\hat{\pi})$ carries $\operatorname{Inn}(\hat{A})$ onto $\operatorname{Inn}(\hat{\pi})$ and is injective (see Section 5.1), it induces an injection $\operatorname{Out}(\hat{A}) \hookrightarrow \operatorname{Out}(\hat{\pi})$. To sum up, we have a sequence of homomorphisms

$$
\mathcal{M}(\Sigma) \stackrel{\zeta}{\longrightarrow} \operatorname{Out}(\pi) \stackrel{\hat{\dot{\zeta}}}{\longrightarrow} \operatorname{Out}(\hat{A}) \hookrightarrow \operatorname{Out}(\hat{\pi})
$$

where the middle map is defined through the obvious extension of automorphisms of $\pi$ to $\hat{A}$. Though it is not important for the sequel, note our belief that this map is injective. Thus we obtain a (presumably injective) group homomorphism $\hat{\zeta}: \mathcal{M}(\Sigma) \rightarrow \operatorname{Out}(\hat{A})$.

The group $\operatorname{Out}(\hat{A})$ does not depend on the choice of the base point $*$ up to canonical isomorphism. Indeed, any path $\gamma:[0,1] \rightarrow \Sigma$ determines an isomorphism $\pi_{1}(\Sigma, \gamma(0)) \simeq \pi_{1}(\Sigma, \gamma(1))$ and an isomorphism of the corresponding completed group algebras. The induced isomorphism of the outer automorphism groups does not depend on $\gamma$.

9.2. Generalized Dehn twists. We define here generalized Dehn twists for closed curves in $\Sigma$. Take a closed disk $D \subset \Sigma$ and provide the surface $\Sigma_{\circ}=\Sigma \backslash \operatorname{Int}(D)$ with orientation induced by that of $\Sigma$. Fix a base point $* \in \partial D \subset \partial \Sigma_{\circ}$. The inclusion homomorphism $\pi_{\circ}=\pi_{1}\left(\Sigma_{\circ}, *\right) \rightarrow \pi_{1}(\Sigma, *)=\pi$ induces an algebra homomorphism $i: A_{\circ}=\mathbb{K}\left[\pi_{\circ}\right] \rightarrow \mathbb{K}[\pi]=A$ and an algebra homomorphism $\hat{i}: \hat{A}_{\circ} \rightarrow \hat{A}$ of the fundamental completions.

Lemma 9.1. Let $\sigma_{\circ}: A_{\circ} \times A_{\circ} \rightarrow A_{\circ}$ be the derived form of the homotopy intersection form of $\Sigma_{\circ}$, and let $\hat{\sigma}_{\circ}: \hat{A}_{\circ} \times \hat{A}_{\circ} \rightarrow \hat{A}_{\circ}$ be the completion of $\sigma_{\circ}$. There is a unique $\mathbb{K}$-bilinear map $\hat{\sigma}: \hat{A}_{\circ} \times \hat{A} \rightarrow \hat{A}$ such that the following diagram commutes:

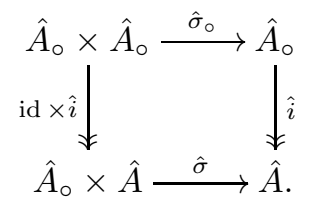

Proof. The uniqueness of $\hat{\sigma}$ is clear because the vertical arrows are surjective. To prove the existence, set $\nu=[\partial D] \in \pi_{\circ}$. It follows from Lemma 7.2 that $\sigma_{\circ}(a, \nu)=0$ for all $a \in \pi_{\circ}$. For any $a, b, c \in \pi_{\circ}$,

$$
\begin{aligned}
\sigma_{\circ}\left(a, b c \nu c^{-1}\right) & =\sigma_{\circ}(a, b) c \nu c^{-1}+b \sigma_{\circ}(a, c) \nu c^{-1}+0+b c \nu \sigma_{\circ}\left(a, c^{-1}\right) \\
& =\sigma_{\circ}(a, b) c \nu c^{-1}+b \sigma_{\circ}(a, c) \nu c^{-1}-b c \nu c^{-1} \sigma_{\circ}(a, c) c^{-1} .
\end{aligned}
$$

Hence $i \sigma_{\circ}\left(a, b c \nu c^{-1}\right)=i \sigma_{\circ}(a, b)$. Since the inclusion homomorphism $\pi_{\circ} \rightarrow \pi$ is surjective and its kernel is normally generated by $\nu$, there is a unique $\mathbb{K}$-bilinear form $\sigma: A_{\circ} \times A \rightarrow A$ such that $i \sigma_{\circ}=\sigma($ id $\times i): A_{\circ} \times A_{\circ} \rightarrow A$. The form $\sigma$ inherits 
the properties of $\sigma_{\circ}$. In particular, Lemma 2.4 implies that $\sigma\left(I_{\circ}^{m}, I^{n}\right) \subset I^{m+n-2}$ for any integers $m, n \geq 1$ where $I_{\circ}$ (resp. $I$ ) is the fundamental ideal of $A_{\circ}$ (resp. $A$ ). Thus, $\sigma$ induces a $\mathbb{K}$-bilinear form $\hat{\sigma}: \hat{A}_{\circ} \times \hat{A} \rightarrow \hat{A}$. The latter satisfies the conditions of the lemma.

The next lemma directly follows from the definitions and the previous lemma.

Lemma 9.2. For any $c \in \hat{\pi}_{\circ}$ and $k \in \mathbb{K}$, the $\mathbb{K}$-linear endomorphism $\hat{\sigma}\left(k \log ^{2}(c),-\right)$ of $\hat{A}$ is a weakly nilpotent derivation of $\hat{A}$, and its exponential $\mathrm{e}^{\hat{\sigma}\left(k \log ^{2}(c),-\right)}$ is an $H$-automorphism of $\hat{A}$ making the following diagram commutative:

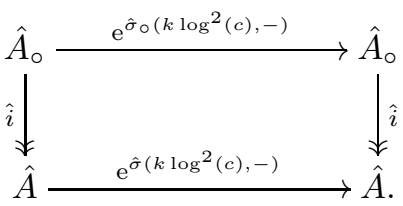

Let now $C$ be a closed curve in $\Sigma$ and $k \in \mathbb{K}$. Pick any $c \in \pi_{\circ}$ such that the conjugacy class of $i(c) \in \pi$ is represented by $C$ (with some orientation). The generalized Dehn twist about $C$ with parameter $k$ is the outer automorphism $\tau_{k, C} \in$ $\operatorname{Out}(\hat{A})$ represented by the H-automorphism $\mathrm{e}^{\hat{\sigma}\left(k \log ^{2}(c),-\right)}$ of $\hat{A}$.

Lemma 9.3. $\tau_{k, C}$ does not depend on the intermediate choices in its definition.

The proof, given in Section 9.4 uses the results of Section 9.3. The properties of $\tau_{k, C}$ are analogous to (and follow from) the properties of the generalized Dehn twists in Section 8. In particular, $\tau_{k, C}$ is a homotopy invariant of $C$.

Theorem 9.4. If $C \subset \Sigma$ is a simple closed curve, then $\tau_{1 / 2, C}=\hat{\zeta}\left(T_{C}\right)$ where $\hat{\zeta}: \mathcal{M}(\Sigma) \rightarrow \operatorname{Out}(\hat{A})$ is introduced in Section 9.1 and $T_{C} \in \mathcal{M}(\Sigma)$ is the classical Dehn twist about $C$.

This theorem follows from the previous lemmas and Theorem 8.3

9.3. Biderivations of $\hat{A}$. A biderivation of $\hat{A}$ is a $\mathbb{K}$-linear endomorphism of $\hat{A}$ which is a derivation in the sense of Section 2.1 and a coderivation in the sense of Lemma 5.5. If a biderivation $\delta$ of $\hat{A}$ is weakly nilpotent, then $\mathrm{e}^{\delta}$ is a welldefined H-automorphism of $\hat{A}$. For example, given $p \in \hat{A}$, consider the derivation $\operatorname{ad}_{p}: \hat{A} \rightarrow \hat{A}$ defined by $\operatorname{ad}_{p}(a)=[p, a]=p a-a p$ for $a \in \hat{A}$. If $p \in \mathcal{P}(\hat{A})$, i.e., if $\hat{\Delta}(p)=p \hat{\otimes} 1+1 \hat{\otimes} p$, then it is easy to check that $\operatorname{ad}_{p}$ is a biderivation. Moreover, $\operatorname{ad}_{p}$ is weakly nilpotent because $\mathcal{P}(\hat{A}) \subset \hat{A}_{1}$ and therefore $\operatorname{ad}_{p}\left(\hat{A}_{i}\right) \subset \hat{A}_{i+1}$ for all $i \geq 0$. The exponential $\mathrm{e}^{\operatorname{ad}_{p}}: \hat{A} \rightarrow \hat{A}$ is an inner automorphism of $\hat{A}$ : it carries any $a \in \hat{A}$ to $\mathrm{e}^{p} a \mathrm{e}^{-p}$ (see Appendix A) and $\mathrm{e}^{p} \in \hat{\pi}$ by (3.2.1).

Lemma 9.5. Let $\delta$ be a weakly nilpotent biderivation of $\hat{A}$. For any $p \in \mathcal{P}(\hat{A})$, the homomorphism $\delta_{p}=\delta+\operatorname{ad}_{p}: \hat{A} \rightarrow \hat{A}$ is a weakly nilpotent biderivation and $\mathrm{e}^{\delta_{p}} \circ\left(\mathrm{e}^{\delta}\right)^{-1}: \hat{A} \rightarrow \hat{A}$ is an inner automorphism of $\hat{A}$.

Proof. Since $\delta$ and $\operatorname{ad}_{p}$ are biderivations, so is $\delta_{p}=\delta+\operatorname{ad}_{p}$. Both $\delta$ and $\operatorname{ad}_{p}$ carry $\hat{A}_{m}$ to $\hat{A}_{m}$ for all $m$, and so does $\delta_{p}$. To show that $\delta_{p}$ is weakly nilpotent, it remains to check that for any $m \geq 0$, a certain power of $\delta_{p}$ carries $\hat{A}$ into $\hat{A}_{m}$. Since $\delta$ is weakly nilpotent, there is an integer $N_{m} \geq 0$ such that $\delta^{N_{m}}(\hat{A}) \subset \hat{A}_{m}$. Observe that any monomial in $\delta, \operatorname{ad}_{p}$ of total degree $\geq m N_{m}$ contains $\geq m$ entries of $\operatorname{ad}_{p}$ 
or $\geq N_{m}$ consecutive entries of $\delta$. Such a monomial carries $\hat{A}=\hat{A}_{0}$ to $\hat{A}_{m}$ because each entry of $\operatorname{ad}_{p}$ increases the filtration degree by 1 and $\delta^{N_{m}}(\hat{A}) \subset \hat{A}_{m}$. Therefore $\delta_{p}^{m N_{m}}(\hat{A}) \subset \hat{A}_{m}$ for all $m$.

Consider the algebra of formal power series $\mathbb{K}\langle\langle x, y\rangle\rangle$ in two non-commuting variables $x, y$. We claim that there is an algebra homomorphism

$$
\mathbb{K}\langle\langle x, y\rangle\rangle \longrightarrow \operatorname{Hom}_{\mathbb{K}}(\hat{A}, \hat{A}), u \longmapsto P_{u}
$$

such that $P_{x}=\delta$ and $P_{y}=\operatorname{ad}_{p}$. This homomorphism carries any $u \in \mathbb{K}\langle\langle x, y\rangle\rangle$ to $P_{u}=\sum_{n \geq 0} u_{n}\left(\delta, \operatorname{ad}_{p}\right)$ where $u_{n}$ denotes the homogeneous degree $n$ part of $u$. We need only to check that, given an integer $m \geq 0$, we have $u_{n}\left(\delta, \operatorname{ad}_{p}\right)(\hat{A}) \subset \hat{A}_{m}$ for all sufficiently big $n$. As above, any monomial in $x, y$ of total degree $\geq m N_{m}$ contains $\geq m$ entries of $y$ or $\geq N_{m}$ consecutive entries of $x$. Therefore the image of such a monomial under (9.3.1) carries $\hat{A}$ to $\hat{A}_{m}$.

Set $\ell=\log \left(\mathrm{e}^{x+y} \mathrm{e}^{-x}\right) \in \mathbb{K}\langle\langle x, y\rangle\rangle$. By the Baker-Campbell-Hausdorff formula,

$$
\ell=(x+y)+(-x)+\frac{1}{2}[x+y,-x]+\cdots=y+\frac{1}{2}[x, y]+\cdots
$$

is a series of Lie polynomials. Clearly, $\mathrm{e}^{x+y}=\mathrm{e}^{\ell} \mathrm{e}^{x}$. Applying $P$, we obtain

$$
\mathrm{e}^{\delta_{p}}=\mathrm{e}^{\delta+\operatorname{ad}_{p}}=\mathrm{e}^{P_{\ell}} \mathrm{e}^{\delta} .
$$

The claim of the lemma follows now from the fact that $\mathrm{e}^{P_{\ell}}$ is an inner automorphism of $\hat{A}$. To see this, expand

$$
\ell=y+\sum_{n \geq 0} \sum_{i \in R_{n}} k_{n, i}\left[v_{n, i}^{(1)}, \ldots\left[v_{n, i}^{(n)},[x, y]\right] \cdots\right]
$$

where $i$ runs over a finite set of indices $R_{n}$ depending on $n, k_{n, i} \in \mathbb{Q} \subset \mathbb{K}$, and $v_{n, i}^{(j)} \in\{x, y\}$ for $j=1, \ldots, n$. Then

$$
P_{\ell}=\operatorname{ad}_{p}+\sum_{n \geq 0} \sum_{i \in R_{n}} k_{n, i}\left[d_{n, i}^{(1)}, \ldots\left[d_{n, i}^{(n)},\left[\delta, \operatorname{ad}_{p}\right]\right] \cdots\right]
$$

where $d_{n, i}^{(j)}$ is $\delta$ or $\operatorname{ad}_{p}$ depending on whether $v_{n, i}^{(j)}$ is $x$ or $y$. Observe that $\left[d, \operatorname{ad}_{c}\right]=$ $\operatorname{ad}_{d(c)}$ for any derivation $d$ of $\hat{A}$ and $c \in \hat{A}$. Therefore

$$
P_{\ell}=\operatorname{ad}_{p}+\sum_{n \geq 0} \sum_{i \in R_{n}} k_{n, i} \operatorname{ad}_{d_{n, i}^{(1)} \cdots d_{n, i}^{(n)} \delta(p)} .
$$

For any $m \geq 0, n \geq m N_{m}-1$, and $i \in R_{n}$, the monomial $v_{n, i}^{(1)} \cdots v_{n, i}^{(n)} x$ contains $\geq m$ entries of $y$ or $\geq N_{m}$ consecutive entries of $x$. In both cases, $d_{n, i}^{(1)} \cdots d_{n, i}^{(n)} \delta(p) \in \hat{A}_{m}$. We deduce that $P_{\ell}=\operatorname{ad}_{q}$ where

$$
q=p+\sum_{n \geq 0} \sum_{i \in R_{n}} k_{n, i} d_{n, i}^{(1)} \cdots d_{n, i}^{(n)} \delta(p)
$$

is a well-defined element of $\hat{A}$. Observe that all coderivations of $\hat{A}$ carry the $\mathbb{K}$ module $\mathcal{P}(\hat{A})$ into itself. Applying this to the coderivations $d_{n, i}^{(j)}$, we deduce that $q \in \mathcal{P}(\hat{A})$. Hence $\mathrm{e}^{P_{\ell}}=\mathrm{e}^{\operatorname{ad}_{q}}$ is an inner automorphism of $\hat{A}$. 
9.4. Proof of Lemma 9.3. We first check the independence of $\tau_{k, C}$ of the choice of $c \in \pi$. Since the kernel of the inclusion homomorphism $\pi_{\circ} \rightarrow \pi$ is normally generated by $\nu=[\partial D]$, it is enough to prove that for any $a, c \in \pi_{\circ}$,

$$
\mathrm{e}^{\hat{\sigma}\left(k \log ^{2}\left(c a \nu a^{-1}\right),-\right)}=\mathrm{e}^{\hat{\sigma}\left(k \log ^{2}(c),-\right)}(\bmod \operatorname{Inn}(\hat{A})) .
$$

By Lemma 2.5,

$$
\hat{\sigma}\left(k \log ^{2}\left(c a \nu a^{-1}\right),-\right)=\hat{\sigma}\left(a^{-1} k \log ^{2}\left(c a \nu a^{-1}\right) a,-\right)=\hat{\sigma}\left(k \log ^{2}\left(a^{-1} c a \nu\right),-\right)
$$

and, similarly,

$$
\hat{\sigma}\left(k \log ^{2}(c),-\right)=\hat{\sigma}\left(k \log ^{2}\left(a^{-1} c a\right),-\right) .
$$

Therefore we need only to prove that for all $c \in \pi_{\circ}$,

$$
\mathrm{e}^{\hat{\sigma}\left(k \log ^{2}(c \nu),-\right)}=\mathrm{e}^{\hat{\sigma}\left(k \log ^{2}(c),-\right)}(\bmod \operatorname{Inn}(\hat{A})) .
$$

Let $\eta_{\circ}: A_{\circ} \times A_{\circ} \rightarrow A_{\circ}$ be the homotopy intersection form of $\Sigma_{\circ}$. For any $b \in \pi_{\circ}$, we have $\eta_{\circ}(\nu, b)=b-1$ and therefore

$$
\eta_{\circ}(c \nu, b)=\eta_{\circ}(c, b)+c \eta_{\circ}(\nu, b)=\eta_{\circ}(c, b)+c b-c .
$$

We expand $\eta_{\circ}(c, b)=\sum_{x \in \pi_{\circ}} l_{x} x$ where $l_{x} \in \mathbb{K}$. Lemma 5.3 gives

$$
\begin{aligned}
\hat{\sigma}_{\circ}\left(k \log ^{2}(c \nu), b\right) & =\sum_{x \in \pi_{\circ}} 2 k b l_{x} x^{-1} \log (c \nu) x+2 k c^{-1} \log (c \nu) c b-2 k b c^{-1} \log (c \nu) c \\
& =\sum_{x \in \pi_{\circ}} 2 k b l_{x} x^{-1} \log (c \nu) x+2 k[\log (\nu c), b] .
\end{aligned}
$$

Applying $\hat{i}: \hat{A}_{\circ} \rightarrow \hat{A}$ and setting $c^{\prime}=i(c) \in \pi$, we obtain

$$
\hat{\sigma}\left(k \log ^{2}(c \nu), i(b)\right)=\sum_{x \in \pi_{\circ}} 2 k l_{x} i\left(b x^{-1}\right) \log \left(c^{\prime}\right) i(x)+2 k\left[\log \left(c^{\prime}\right), i(b)\right] .
$$

A similar computation gives

$$
\hat{\sigma}\left(k \log ^{2}(c), i(b)\right)=\sum_{x \in \pi_{\circ}} 2 k l_{x} i\left(b x^{-1}\right) \log \left(c^{\prime}\right) i(x) .
$$

Thus, the weakly nilpotent biderivations $\hat{\sigma}\left(k \log ^{2}(c \nu),-\right)$ and $\hat{\sigma}\left(k \log ^{2}(c),-\right)$ of $\hat{A}$ differ by $\left[2 k \log \left(c^{\prime}\right),-\right]$. Since $2 k \log \left(c^{\prime}\right) \in \mathcal{P}(\hat{A})$, Lemma 9.5 implies (9.4.1).

To finish the proof, we need to show the independence of $\tau_{k, C}$ of the choice of $D$ and $* \in \partial D$. If $D^{+} \subset \operatorname{Int}(D)$ is a smaller 2-disk with pointed boundary, then $\Sigma_{\circ}=\Sigma \backslash \operatorname{Int}(D)$ is a subsurface of $\Sigma_{\circ}^{+}=\Sigma \backslash \operatorname{Int}\left(D^{+}\right)$and the required result follows from the naturality of the twists (8.3.1) and Lemma 9.2. Passing in this way from a disk in $\Sigma$ to a smaller (or a bigger) disk we can relate any two embedded disks in $\Sigma$ with pointed boundary. This implies our claim.

9.5. Remarks. 1. The H-automorphisms of $\hat{A}$ act on $\hat{A}_{1} / \hat{A}_{2} \simeq I / I^{2} \simeq H_{1}(\Sigma ; \mathbb{K})$ in the obvious way. Let Aut. $(\hat{A})$ be the subgroup of $\operatorname{Aut}(\hat{A})$ formed by the Hautomorphisms preserving the homological intersection form in $H_{1}(\Sigma ; \mathbb{K})$. Clearly, $\operatorname{Inn}(\hat{A}) \subset$ Aut. $(\hat{A})$. It is easy to check that for any closed curve $C$ and $k \in \mathbb{K}$, the twist $\tau_{k, C}$ lies in Aut. $(\hat{A}) / \operatorname{Inn}(\hat{A}) \subset \operatorname{Out}(\hat{A})$.

2. If $\partial \Sigma \neq \emptyset$, then the twists $\tau$ constructed in this section can be computed without cutting out a disk from $\Sigma$. For any $* \in \partial \Sigma$, any closed curve $C$ in $\Sigma$, and any $k \in \mathbb{K}$, Section 8 yields a twist $t_{k, C} \in \operatorname{Aut}(\hat{A})$ where $A=\mathbb{K}\left[\pi_{1}(\Sigma, *)\right]$. It can be checked that projecting to $\operatorname{Out}(\hat{A})$ we obtain $\tau_{k, C}$. Note the following consequence: 
the twists $t_{k, C}$ corresponding to different choices of $*$ on $\partial \Sigma$ are obtained from each other through transportation along a path connecting the base points followed by an inner automorphism of the completed group algebra.

\section{Symplectic expansions And the KaWAZumi-Kuno APproach}

In this section $\Sigma$ is a compact connected oriented surface with connected boundary, $* \in \partial \Sigma$, and $\mathbb{K}=\mathbb{Q}$. We outline the work of Kawazumi and Kuno KK1, Ku who first defined generalized Dehn twists for curves in such a $\Sigma$ using symplectic expansions of $\pi=\pi_{1}(\Sigma, *)$. We show that their definition is equivalent to ours.

10.1. Symplectic expansions. Set $H=H_{1}(\pi ; \mathbb{Q})$ and let $T=\oplus_{m \geq 0} H^{\otimes m}$ be the tensor algebra of $H$. The degree completion $\hat{T}=\prod_{m \geq 0} H^{\otimes m}$ of $T$ has the filtration $\hat{T}=\hat{T}_{0} \supset \hat{T}_{1} \supset \hat{T}_{2} \supset \cdots$ where $\hat{T}_{m}=\prod_{n \geq m} H^{\otimes n}$ for all $m \geq 0$. The algebra $\hat{T}$ has a natural comultiplication carrying any $h \in H$ to $h \hat{\otimes} 1+1 \hat{\otimes} h$. This turns $\hat{T}$ in a complete Hopf algebra.

A Magnus expansion of $\pi$ is a monoid homomorphism $\theta: \pi \rightarrow \hat{T}$ such that $\theta(x)=1+[x]\left(\bmod \hat{T}_{2}\right)$ for all $x \in \pi$, where $[x] \in H$ is the homology class of $x$ (see Ka $]$. Such a $\theta$ induces an algebra isomorphism $\hat{\theta}: \hat{A} \rightarrow \hat{T}$ carrying $\hat{A}_{m} \subset \hat{A}$ onto $\hat{T}_{m}$ for all $m \geq 0$. A symplectic expansion of $\pi$ is a Magnus expansion $\theta: \pi \rightarrow \hat{T}$ satisfying two additional conditions: the group-like condition and the symplectic condition (see $\mathrm{Ma}$ ). The former says that all elements of $\theta(\pi)$ are group-like or equivalently that $\hat{\theta}$ preserves comultiplication. To state the symplectic condition, note that the $\mathbb{Q}$-valued intersection form - in $H$ is non-degenerate (here we use that $\Sigma$ is compact and $\partial \Sigma$ is connected). Consider the duality isomorphism

$$
H \stackrel{\simeq}{\longrightarrow} H^{*}=\operatorname{Hom}_{\mathbb{Q}}(H, \mathbb{Q}), h \longmapsto h \cdot(-) .
$$

We use this isomorphism to transform the intersection form $\cdot \in \Lambda^{2} H^{*}$ into an element $\omega$ of $\Lambda^{2} H \subset H^{\otimes 2} \subset T$. The symplectic condition on $\theta$ says that $\hat{\theta}$ carries the homotopy class of the loop $\partial \Sigma$ based at $*$ to $\mathrm{e}^{-\omega}$.

10.2. Symplectic derivations of $\hat{T}$. A derivation $d$ of the algebra $\hat{T}$ is filtered if $d\left(\hat{T}_{m}\right) \subset \hat{T}_{m}$ for all $m \geq 0$. Denote by $\operatorname{Der}(\hat{T})$ the Lie algebra of filtered derivations of $\hat{T}$. Let $\operatorname{Der}_{\omega}(\hat{T})$ be the Lie subalgebra of $\operatorname{Der}(\hat{T})$ consisting of the derivations that vanish on $\omega \in H^{\otimes 2}$. Restricting the derivations in $\hat{T}$ to $H$ and using (10.1.1), we obtain canonical isomorphisms

$$
\operatorname{Der}(\hat{T}) \simeq \operatorname{Hom}\left(H, \hat{T}_{1}\right) \simeq H^{*} \otimes \hat{T}_{1} \simeq H \otimes \hat{T}_{1}
$$

It is known that these isomorphisms carry $\operatorname{Der}_{\omega}(\hat{T})$ onto

$$
D=\operatorname{Ker}\left([-,-]: H \otimes \hat{T}_{1} \longrightarrow \hat{T}_{2}\right) \subset H \otimes \hat{T}_{1} \subset \hat{T}_{2} .
$$

Hence there is an action of $D$ on $\hat{T}$ by filtered derivations vanishing on $\omega$. The Lie algebra $D \simeq \operatorname{Der}_{\omega}(\hat{T})$ was introduced by Kontsevich [Ko], and is sometimes called the Lie algebra of symplectic derivations.

Kawazumi and Kuno [KK1] define a "cyclicization" map $N: \hat{T}_{1} \rightarrow \hat{T}_{1}$ by

$$
N\left(h_{1} \otimes \cdots \otimes h_{m}\right)=\sum_{i=1}^{m} h_{i} \otimes \cdots \otimes h_{m} \otimes h_{1} \otimes \cdots \otimes h_{i-1}
$$


for any $m \geq 1$ and $h_{1}, \ldots, h_{m} \in H$. We have $N\left(\hat{T}_{2}\right) \subset D$ (see KK1). Then $\hat{T}$ acts on itself by derivations (not necessarily filtered) as follows: $\mathbb{Q}=H^{\otimes 0}$ acts as zero; any $h \in H=H^{\otimes 1}$ acts by $k \mapsto h \cdot k$ for $k \in H$; any $t \in \hat{T}_{2}$ acts as $N(t) \in$ $D \simeq \operatorname{Der}_{\omega}(\hat{T})$. For any $a, b \in \hat{T}$, let $\langle a, b\rangle \in \hat{T}$ be the evaluation of the derivation determined by $a$ on $b$. The following theorem uses the pairing $\langle-,-\rangle: \hat{T} \times \hat{T} \rightarrow \hat{T}$ to give a tensorial computation of the pairing $\hat{\sigma}: \hat{A} \times \hat{A} \rightarrow \hat{A}$ obtained as the completion of the form $\sigma: A \times A \rightarrow A$ defined by (7.2.2).

Theorem 10.1 (Kawazumi-Kuno [KK1]). For any symplectic expansion $\theta$ of $\pi$, the following diagram commutes:

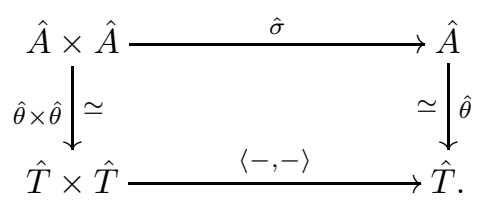

The statement of this theorem in KK1 has a minus sign because the duality used there is minus the duality (10.1.1). We give below a new proof of Theorem 10.1 .

Kuno $\left[\mathrm{Ku}\right.$ ] defines a "generalized Dehn twist" $t_{C}$ about a closed curve $C$ in $\Sigma$ as follows. Let $c \in \pi$ lie in the conjugacy class determined by $C$ (endowed with any orientation). Pick a symplectic expansion $\theta$ of $\pi$ and, following [KK1], consider the derivation

$$
L^{\theta}(c)=\left\langle\log ^{2}(\theta(c)) / 2,-\right\rangle: \hat{T} \longrightarrow \hat{T} .
$$

Then $\mathrm{e}^{L^{\theta}(c)}$ is an algebra automorphism of $\hat{T}$ and $t_{C}=\hat{\theta}^{-1} \mathrm{e}^{L^{\theta}(c)} \hat{\theta}$ is an algebra automorphism of $\hat{A}$. Kuno $\left[\mathrm{Ku}\right.$. shows that $t_{C}$ does not depend on the choice of $\theta$. By KK1, if $C$ is simple, then $t_{C}$ is the automorphism of $\hat{A}$ induced by the classical Dehn twist about $C$. The following lemma establishes the equivalence of the Kawazumi-Kuno approach with ours.

Lemma 10.2. For any closed curve $C$ in $\Sigma$, we have $t_{C}=t_{1 / 2, C}$.

Proof. Theorem 10.1 implies that

$$
\mathrm{e}^{L^{\theta}(c)}=\mathrm{e}^{\left\langle\hat{\theta}\left(\log ^{2}(c) / 2\right),-\right\rangle}=\mathrm{e}^{\hat{\theta} \hat{\sigma}\left(\log ^{2}(c) / 2,-\right) \hat{\theta}^{-1}}=\hat{\theta} \mathrm{e}^{\hat{\sigma}\left(\log ^{2}(c) / 2,-\right)} \hat{\theta}^{-1}
$$

and the conclusion follows.

10.3. The group $\widehat{\mathcal{M}}(\Sigma, *)$. The generalized Dehn twists $t_{k, C}$ of closed curves in $\Sigma$ belong to the extended mapping class group $\widehat{\mathcal{M}}(\Sigma, *)$. By Section 8.1 , this is the group of $\mathrm{H}$-automorphisms of $\hat{A}$ preserving the F-pairing $\hat{\eta}: \hat{A} \times \hat{A} \rightarrow \hat{A}$ obtained as the completion of the homotopy intersection form $\eta$ in $A \subset \hat{A}$. Under the assumptions of this section on $\Sigma$ and $\mathbb{K}$, we can say more about $\widehat{\mathcal{M}}(\Sigma, *)$.

Since $\mathbb{K}=\mathbb{Q}$, the natural homomorphism $\operatorname{Aut}(\hat{A}) \rightarrow \operatorname{Aut}(\hat{\pi})$ is an isomorphism. We claim that this isomorphism carries $\widehat{\mathcal{M}}(\Sigma, *) \subset \operatorname{Aut}(\hat{A})$ onto the $\operatorname{group} \operatorname{Aut}_{\nu}(\hat{\pi})$ consisting of all filtered automorphisms of $\hat{\pi}$ fixing $\nu=[\partial \Sigma] \in \pi \subset \hat{\pi}$. Indeed, our assumptions on $\Sigma$ imply that $\hat{\eta}$ is non-degenerate. The element $\nu$ satisfies $\eta(x, \nu)=x-1$ for all $x \in \pi$. Therefore, in the notation of Section 6.1. $\nabla_{\hat{\eta}}=\nu-1$. Now, our claim directly follows from Lemma 6.3. Thus, $\widehat{\mathcal{M}}(\Sigma, *) \simeq \operatorname{Aut}_{\nu}(\hat{\pi})$. 
10.4. A tensorial description of $\hat{\eta}$. We give a tensorial description of the homotopy intersection form $\hat{\eta}$ in $\hat{A}$. It is used below to prove Theorem 10.1.

Let $\varepsilon: \hat{T} \rightarrow \mathbb{Q}$ be the counit of $\hat{T}$ defined by $\varepsilon(1)=1$ and $\varepsilon\left(H^{\otimes m}\right)=0$ for all $m>0$. A bilinear pairing $\rho: \hat{T} \times \hat{T} \rightarrow \hat{T}$ is an F-pairing if it satisfies the identities (2.2.1) and (2.2.2) with $\eta, A$, aug replaced by $\rho, \hat{T}, \varepsilon$, respectively. This condition may be rewritten as $\rho(1, \hat{T})=\rho(\hat{T}, 1)=0$ and

$$
\begin{aligned}
& \rho\left(a_{1} a_{2}, b\right)=a_{1} \rho\left(a_{2}, b\right) \text { for all } a_{1}, a_{2}, b \in \hat{T}_{1}, \\
& \rho\left(a, b_{1} b_{2}\right)=\rho\left(a, b_{1}\right) b_{2} \text { for all } a, b_{1}, b_{2} \in \hat{T}_{1} .
\end{aligned}
$$

An F-pairing $\rho: \hat{T} \times \hat{T} \rightarrow \hat{T}$ is filtered if for all integer $m \geq 2$,

$$
\rho\left(\hat{T}_{m}, \hat{T}\right) \subset \hat{T}_{m-1} \supset \rho\left(\hat{T}, \hat{T}_{m}\right) .
$$

Denote by $\dot{\rightsquigarrow}$ the bilinear map $\hat{T}_{1} \times \hat{T}_{1} \rightarrow \hat{T}$ defined on $H^{\otimes m} \times H^{\otimes n}$ (for all $m, n>0)$ by a single contraction:

$$
\left(h_{1} \otimes \cdots \otimes h_{m} \dot{\rightsquigarrow} k_{1} \otimes \cdots \otimes k_{n}\right)=\left(h_{m} \cdot k_{1}\right) h_{1} \otimes \cdots \otimes h_{m-1} \otimes k_{2} \otimes \cdots \otimes k_{n}
$$

where $\cdot$ is the homological intersection form in $H$. Using the formal power series

$$
s(z)=\frac{1}{\mathrm{e}^{-z}-1}+\frac{1}{z}=-\frac{1}{2}-\sum_{k \geq 1} \frac{B_{2 k}}{(2 k) !} z^{2 k-1}=-\frac{1}{2}-\frac{z}{12}+\frac{z^{3}}{720}-\frac{z^{5}}{30240}+\cdots
$$

we define a bilinear map $\rho: \hat{T} \times \hat{T} \rightarrow \hat{T}$ by

$$
\rho(a, b)=(a-\varepsilon(a)) \dot{\rightsquigarrow}(b-\varepsilon(b))+(a-\varepsilon(a)) s(\omega)(b-\varepsilon(b))
$$

for all $a, b \in \hat{T}$. Here $\omega$ is the element of $\Lambda^{2} H$ dual to the intersection form.

Lemma 10.3. The form $\rho$ is a filtered F-pairing in $\hat{T}$.

Proof. Clearly, $\rho(a, b)=0$ whenever $a=1$ or $b=1$ and, for all $a, b \in \hat{T}_{1}$,

$$
\rho(a, b)=a \dot{\rightsquigarrow} b+a s(\omega) b .
$$

This easily implies the claim of the lemma.

Theorem 10.4. Let $\theta: \pi \rightarrow \hat{T}$ be a Magnus expansion of $\pi$ satisfying the symplectic condition. Then the following diagram is commutative:

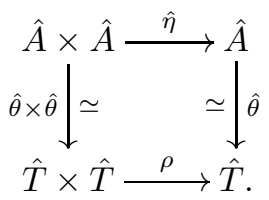

Proof. Since the algebra isomorphism $\hat{\theta}: \hat{A} \rightarrow \hat{T}$ induced by $\theta$ preserves the counit and the filtration, $\rho_{\theta}=\hat{\theta}^{-1} \rho(\hat{\theta} \times \hat{\theta}): \hat{A} \times \hat{A} \rightarrow \hat{A}$ is a filtered F-pairing. It is non-degenerate in the sense of Section 6.1 because $\pi$ is finitely generated and for all $x, y \in \pi$,

$$
\text { aûg } \begin{aligned}
\rho_{\theta}(x, y) & =\varepsilon \rho(\theta(x), \theta(y)) \\
& =\varepsilon((\theta(x)-1) \dot{\rightsquigarrow}(\theta(y)-1))=[x] \cdot[y] .
\end{aligned}
$$


As observed above, $\nabla_{\hat{\eta}}=\nu-1$. The theorem will follow from Lemma 6.2 as soon as we show that $\nabla_{\rho_{\theta}}=\nu-1$ as well. By the symplectic condition on $\theta$, this is equivalent to $\rho\left(a, \mathrm{e}^{-\omega}\right)=a-\varepsilon(a)$ for all $a \in \hat{T}$. It is enough to prove the latter for $a=h \in H$. In this case,

$$
\begin{aligned}
\rho\left(h, \mathrm{e}^{-\omega}\right) & =\sum_{r \geq 1} \frac{(-1)^{r}}{r !} \rho(h, \omega) \omega^{r-1} \\
& =\rho(h, \omega) \frac{\mathrm{e}^{-\omega}-1}{\omega} \\
& =(h \dot{\rightsquigarrow} \omega+h s(\omega) \omega) \frac{\mathrm{e}^{-\omega}-1}{\omega} \\
& =h(-1+s(\omega) \omega) \frac{\mathrm{e}^{-\omega}-1}{\omega}=h .
\end{aligned}
$$

Here we use that $h \dot{\rightsquigarrow} \omega=-h$; this is checked using a symplectic basis of $H$.

10.5. Proof of Theorem 10.1. The following lemma is an easy consequence of the definition of the pairing $\langle-,-\rangle$ in $\hat{T}$.

Lemma 10.5. For any $h_{1}, \ldots, h_{m}, k_{1}, \ldots, k_{n} \in H$,

$$
\left\langle h_{1} \cdots h_{m}, k_{1} \cdots k_{n}\right\rangle=-\sum_{j=1}^{n} k_{1} \cdots k_{j-1}\left(k_{j} \dot{\rightsquigarrow} N\left(h_{1} \cdots h_{m}\right)\right) k_{j+1} \cdots k_{n} .
$$

Let now $\theta$ be a symplectic expansion of $\pi$. Set

$$
\sigma^{\prime}=\hat{\theta} \hat{\sigma}\left(\hat{\theta}^{-1} \times \hat{\theta}^{-1}\right): \hat{T} \times \hat{T} \longrightarrow \hat{T} .
$$

We should show that $\sigma^{\prime}=\langle-,-\rangle$. Let $\eta_{-}$be the F-pairing in $\hat{A}$ defined by

$$
\eta_{-}(a, b)=\hat{\eta}(a, b)-(a-\operatorname{aûg}(a)) s(-\log (\nu))(b-\operatorname{aûg}(b)) \in \hat{A},
$$

for all $a, b \in \hat{A}$. This F-pairing is equivalent to $\hat{\eta}$ in the sense of Section 2.4 (where we extend the terminology of that section to F-pairings in $\hat{A}$ in the obvious way). Therefore $\eta_{-}$and $\hat{\eta}$ have the same derived form: $\hat{\sigma}^{\eta_{-}}=\hat{\sigma}^{\hat{\eta}}=\widehat{\sigma^{\eta}}=\hat{\sigma}: \hat{A} \times \hat{A} \rightarrow \hat{A}$. Lemma 3.4 computes $\hat{\sigma}$ from $\eta_{-}$. Since $\hat{\theta}: \hat{A} \rightarrow \hat{T}$ is a filtration-preserving Hopf algebra isomorphism, we deduce that

$$
\sigma^{\prime}=\mu(\mu \hat{\otimes} \mu) P_{4213}\left(\operatorname{id}_{\hat{T}} \hat{\otimes}\left(S \hat{\otimes} \operatorname{id}_{\hat{T}}\right) \Delta \eta_{-}^{\prime} \hat{\otimes} \operatorname{id}_{\hat{T}}\right)(\Delta \hat{\otimes} \Delta),
$$

where $\mu, \Delta$, and $S$ are multiplication, comultiplication, and antipode in $\hat{T}$, and

$$
\eta_{-}^{\prime}=\hat{\theta} \eta_{-}\left(\hat{\theta}^{-1} \times \hat{\theta}^{-1}\right): \hat{T} \times \hat{T} \longrightarrow \hat{T} .
$$

Theorem 10.4 implies that for all $c, d \in \hat{T}$,

$$
\eta_{-}^{\prime}(c, d)=(c-\varepsilon(c)) \dot{\rightsquigarrow}(d-\varepsilon(d)) .
$$

Hence $\eta_{-}^{\prime}$ is a map of degree -2 . Since $\mu, \Delta, S$ are degree 0 maps, $\sigma^{\prime}$ is a degree -2 map. So, $\sigma^{\prime}\left(h_{1} \cdots h_{m}, k_{1} \cdots k_{n}\right) \in H^{\otimes(m+n-2)}$ for any $h_{1}, \ldots, h_{m}, k_{1}, \ldots, k_{n} \in H$. Lemma 2.4 implies that $\sigma^{\prime}\left(h_{1} \cdots h_{m}, k_{1} \cdots k_{n}\right)$ is congruent modulo $\hat{T}_{m+n-1}$ to

$$
\sum_{i=1}^{m} \sum_{j=1}^{n}\left(h_{i} \cdot k_{j}\right) k_{1} \cdots k_{j-1}\left(h_{i+1} \cdots h_{m} h_{1} \cdots h_{i-1}\right) k_{j+1} \cdots k_{n} .
$$


We deduce that this congruence is actually an equality in $\hat{T}$. Comparing with Lemma 10.5, we conclude that $\sigma^{\prime}\left(h_{1} \cdots h_{m}, k_{1} \cdots k_{n}\right)=\left\langle h_{1} \cdots h_{m}, k_{1} \cdots k_{n}\right\rangle$. This is equivalent to the claim of the theorem.

\section{Appendix A. Formal identities}

We gather here a few classical formulas used in the main body of the paper. These formulas hold in the ring of formal power series in $n$ non-commuting variables $P=\mathbb{K}\left\langle\left\langle x_{1}, \ldots, x_{n}\right\rangle\right\rangle$ with coefficients in a commutative ring $\mathbb{K} \supset \mathbb{Q}$. Consider the degree filtration $P=P_{0} \supset P_{1} \supset P_{2} \supset \cdots$. For $u \in P_{1}$, set

$$
\mathrm{e}^{u}=\sum_{k \geq 0} \frac{u^{k}}{k !} \in 1+P_{1} \quad \text { and } \quad \log (1+u)=\sum_{k \geq 1}(-1)^{k+1} \frac{u^{k}}{k} \in P_{1} .
$$

The obvious identities $\mathrm{e}^{\log (1+u)}=1+u$ and $\log \left(\mathrm{e}^{u}\right)=u$ imply that the exponential map and the logarithm are mutually inverse bijections:

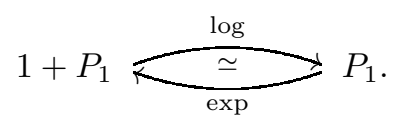

The Baker-Campbell-Hausdorff formula asserts that for all $u, v \in P_{1}$,

$$
\log \left(\mathrm{e}^{u} \mathrm{e}^{v}\right)=u+v+\frac{1}{2}[u, v]+\frac{1}{12}[u,[u, v]]+\frac{1}{12}[v,[v, u]]+\cdots,
$$

where the dots stand for a series of Lie polynomials in $u, v$ of degree $\geq 4$ (see, for instance, MKS, Theorem 5.19). In particular, if $u, v \in P_{1}$ commute, then $\mathrm{e}^{u} \mathrm{e}^{v}=\mathrm{e}^{u+v}$. Also, $\log \left((1+u)^{m}\right)=m \log (1+u)$ for any $u \in P_{1}$ and $m \in \mathbb{Z}$. For all $u \in P_{1}$ and $v \in 1+P_{1}$,

$$
v \log (1+u) v^{-1}=\log \left(v(1+u) v^{-1}\right) .
$$

Finally, we mention the following formula (see Exercice 5.9.7 of [MKS]): for all $u \in P_{1}$ and $v \in P$,

$$
\mathrm{e}^{u} v \mathrm{e}^{-u}=\sum_{n \geq 0} \frac{\overbrace{u,[u, \cdots[u}^{n \text { times }}, v] \cdots]]}{n !}=\mathrm{e}^{[u,-]}(v) .
$$

Using the universal property of $P=\mathbb{K}\left\langle\left\langle x_{1}, \ldots, x_{n}\right\rangle\right\rangle$, one can obtain similar formulas in other settings. For example, consider a complete augmented algebra $R$ in the sense of $\mathrm{Qu}$ : thus, $R$ is a $\mathbb{K}$-algebra with a filtration by submodules $R=R_{0} \supset R_{1} \supset R_{2} \supset \cdots$ such that $R_{i} R_{j} \subset R_{i+j}$ for all $i, j \geq 0$, the $\mathbb{K}$-algebra $R / R_{1}$ is isomorphic to $\mathbb{K}$ and the canonical map $R \rightarrow \varliminf_{\swarrow} R / R_{m}$ is an isomorphism. (Such $R$ arise in this paper as the fundamental completions of group algebras.) Formulas (A.0.1) define the mutually inverse logarithm $1+R_{1} \rightarrow R_{1}$ and exponent $R_{1} \rightarrow 1+R_{1}$. The identities in $P$ stated above give rise to similar identities in $R$. For example, for all $r \in R_{1}$ and $s \in R$,

$$
\mathrm{e}^{r} s \mathrm{e}^{-r}=\sum_{n \geq 0} \frac{[r,[r, \cdots[r, s] \cdots]]}{n !}=\mathrm{e}^{[r,-]}(s) .
$$

This is obtained by assuming that $s \in R_{1}$ (which is allowed since $R=\mathbb{K} \cdot 1+R_{1}$ ), applying A.0.2 to $u=x_{1}$, and $v=x_{2}$ and transporting the resulting equality to $R$ via the algebra homomorphism $\mathbb{K}\left\langle\left\langle x_{1}, x_{2}\right\rangle\right\rangle \rightarrow R, x_{1} \mapsto r, x_{2} \mapsto s$. 


\section{REFERENCES}

[Ep] D. B. A. Epstein, Curves on 2-manifolds and isotopies. Acta Math. 115 (1966), 83-107.

[GL] S. Garoufalidis, J. Levine, Tree-level invariants of three-manifolds, Massey products and the Johnson homomorphism. Graphs and patterns in mathematics and theoretical physics, 173-203, Proc. Sympos. Pure Math., 73, Amer. Math. Soc., Providence, RI, 2005.

[Go] W. M. Goldman, Invariant functions on Lie groups and Hamiltonian flows of surface group representations. Invent. Math. 85 (1986), no. 2, 263-302.

[Ha] N. Habegger, Milnor, Johnson and the tree-level perturbative invariants. Preprint (2000), University of Nantes.

[Je] S. A. Jennings, The group ring of a class of infinite nilpotent groups. Canad. J. Math. 7 (1955), 169-187.

[Ka] N. Kawazumi, Cohomological aspects of Magnus expansions. Preprint (2005) arXiv:math/0505497.

[KK1] N. Kawazumi, Y. Kuno, The logarithms of Dehn twists. Preprint (2010) arXiv: 1008.5017.

[KK2] N. Kawazumi, Y. Kuno, Groupoid-theoretical methods in the mapping class groups of surfaces. Preprint (2011) arXiv:1109.6479.

[Ko] M. Kontsevich, Formal (non) commutative symplectic geometry. The Gel'fand Mathematical Seminars, 1990-1992, 173-187, Birkhäuser Boston, Boston, MA, 1993.

$[\mathrm{Ku}] \quad Y$. Kuno, The generalized Dehn twist along a figure eight. Preprint (2011) arXiv: 1104.2107.

[MKS] W. Magnus, A. Karrass, D. Solitar, Combinatorial group theory. Presentations of groups in terms of generators and relations. Second revised edition. Dover Publications, Inc., New York, 1976.

[Ma] G. Massuyeau, Infinitesimal Morita homomorphisms and the tree-level of the LMO invariant. Preprint (2008) arXiv:0809.4629.

[Mo] S. Morita, Symplectic automorphism groups of nilpotent quotients of fundamental groups of surfaces. Groups of diffeomorphisms, 443-468, Adv. Stud. Pure Math., 52, Math. Soc. Japan, Tokyo, 2008,

[Pa] C. D. Papakyriakopoulos, Planar regular coverings of orientable closed surfaces. Knots, groups, and 3-manifolds (Papers dedicated to the memory of R. H. Fox), 261-292. Ann. of Math. Studies, No. 84, Princeton Univ. Press, Princeton, N.J., 1975.

[Pe] B. Perron, A homotopic intersection theory on surfaces: applications to mapping class group and braids. Enseign. Math. (2) 52 (2006), no. 1-2, 159-186.

[Qu] D. Quillen, Rational homotopy theory. Ann. of Math. (2) 90 (1969), 205-295.

[Tu1] V. G. Turaev, Intersections of loops in two-dimensional manifolds. (Russian) Mat. Sb. 106(148) (1978), 566-588. English translation: Math. USSR, Sb. 35 (1979), 229-250.

[Tu2] V. G. Turaev, Multiplace generalizations of the Seifert form of a classical knot. (Russian) Mat. Sb. 116(158) (1981), 370-397. English translation: Math. USSR, Sb. 44 (1983), $335-361$.

GwénaëL Massuyeau

IRMA, Université de Strasbourg \& CNRS

7 rue René Descartes

67084 Strasbourg, France

massuyeau@math.unistra.fr

Vladimir Turaev

Department of MATHEMATics

INDIANA UNIVERSITY

BLOOMINGTON IN47405, USA

and

IRMA, Université de Strasbourg \& CNRS

7 RUE RENÉ DESCARTES

67084 Strasbourg, France

vturaev@yahoo.com 